\title{
A Lattice Formulation of Super Yang-Mills Theories with Exact Supersymmetry
}

\author{
Fumihiko Sugino \\ School of Physics 83 BK-21 Physics Division \\ Seoul National University, Seoul 151-747, Korea \\ E-mail: sugino@phya.snu.ac.kr
}

\begin{abstract}
We construct super Yang-Mills theories with extended supersymmetry on hypercubic lattices of various dimensions keeping one or two supercharges exactly. Gauge fields are represented by ordinary unitary link variables, and the exact supercharges are nilpotent up to gauge transformations. Among the models, we show that the desired continuum theories are obtained without any fine tuning of parameters for the cases $\mathcal{N}=2,4,8$ in two-dimensions.
\end{abstract}

KeYwords: Lattice Quantum Field Theory, Lattice Gauge Field Theories, Extended Supersymmetry, Topological Field Theories. 


\section{Contents}

1. Introduction 1

2. 'Topological Field Theory Form' of $\mathcal{N}=2$ Super Yang-Mills Theories 4

2.1 Note on Wick-rotation and $\mathrm{U}(1)_{R}$ Symmetry 4

2.2 'Topological Field Theory Form' 5

3. Lattice Formulation with One Exact Supercharge $\quad 7$

$\begin{array}{lll}3.1 & \text { Lattice Action } & 7\end{array}$

3.2 Absence of Fermion Doubling 9

3.3 Renormalization 11

4. 'Balanced Topological Field Theory Form' of $\mathcal{N}=4$ Super Yang-Mills Theories $\quad 12$

4.1 Two-dimensional Case 12

$\begin{array}{lll}4.2 & \text { Four-dimensional Case } & 14\end{array}$

5. Lattice Formulation with Two Exact Supercharges $\quad 15$

$\begin{array}{lll}5.1 & \text { Two-dimensional Case } & 17\end{array}$

$\begin{array}{lll}5.2 & \text { Four-dimensional Case } & 18\end{array}$

$\begin{array}{ll}5.3 \mathcal{N}=8 \text { in Three-dimensions } & 19\end{array}$

$5.4 \mathcal{N}=8$ in Two-dimensions 20

6. Summary and Discussions $\quad 21$

A. Explicit Form of $\gamma_{i}$ for $\mathcal{N}=2 \quad 22$

B. Explicit Form of $P_{\mu}$ for $\mathcal{N}=2,4$

C. $Q_{ \pm}$-cohomology on Lattice $\quad 24$

\section{Introduction}

Nonperturbative aspects in supersymmetric gauge theory have been vigorously investigated, in particular since finding the exact form of the low energy effective action in fourdimensional $\mathcal{N}=2$ super Yang-Mills theory [1] and the AdS/CFT duality between gauge theory and gravity (string theory) [2]. For some operators such as the BPS saturated objects or the chiral operators, which are protected from the quantum corrections by certain 
algebraic properties, we can know exact results for their spectra or the correlation functions. The approach to the nonperturbative physics is based on the property of duality.

A conventional but more universal approach is lattice formulation, which enables numerical analysis for any observables not restricted to special operators with the BPS saturated or chiral properties. However, there has been difficulty on the lattice approach to supersymmetry, because of lack of infinitesimal translational invariance on the lattice and breakdown of the Leibniz rule [3, 4]. In spite of the difficulty, it is possible to construct lattice models, which do not have manifest supersymmetry but flow to the desired supersymmetric theories in the continuum limit. One of the examples is $\mathcal{N}=1$ super Yang-Mills (SYM) theory in four-dimensions whose field contents are gauge bosons and gauginos. Since in the theory the only relevant supersymmetry breaking operator is the gaugino mass, one can arrive at the supersymmetric continuum theory if the radiative corrections are not allowed to induce the relevant operator by symmetries realized in the lattice theory. Making use of domain wall or overlap fermions keeps discrete chiral symmetry on lattice, which is the symmetry excluding the fermion mass [5].

Supersymmetric theories with extended supersymmetry have some supercharges, which are not related to the infinitesimal translations and can be seen as fermionic internal symmetries. It is possible to realize a part of such supercharges as exact symmetry on lattice, and the exact supersymmetry plays a key role to restore the full supersymmetry in the continuum limit without any fine tuning of parameters. One of the examples is $\mathcal{N}=2$ Wess-Zumino model in two-dimensions where the Nicolai mapping was heavily utilized to construct the lattice model [6]. Also for SYM theories with extended supersymmetry, Cohen, Kaplan, Katz and Ünsal recently proposed such a kind of various lattice models motivated by the idea of deconstruction $[7,8]^{1}$. In these models, to generate the kinetic terms of the target theories and to stabilize noncompact bosonic zero-modes (the so-called radions), one has to add terms softly breaking the exact supersymmetry, which are tuned to vanish in large volume limit.

In many cases, the above 'internal' supersymmetries can be reinterpreted as the BRST symmetries in topological field theories. So, in order to construct lattice models respecting the 'internal' supersymmetry, it seems natural to start with the topological field theory formulation of the theory. In ref. [11], Catterall discussed on a general formulation of lattice models based on the connection to topological field theory for supersymmetric theories without gauge symmetry. Two-dimensional $\mathcal{N}=2$ Wess-Zumino model was also investigated there.

Here, standing on the same philosophy, we construct lattice models for SYM theories with extended supersymmetry keeping one or two supercharges exactly. Our models are motivated by the topological field theory formulation of $\mathcal{N}=2,4 \mathrm{SYM}$ theories, and free from the radion problems. The lattices have hypercubic structures, and the gauge fields are expressed as ordinary compact unitary variables on the lattice links. Our lattice models have a huge degeneracy of the classical vacua growing as exponential of the number of plaquettes, which causes difficulty for perturbative analysis around a specific vacuum.

\footnotetext{
${ }^{1}$ For some related works, see refs. [9]. Also, for other attempts to lattice formulations of supersymmetry, see $[10,11]$.
} 
From the reason not completely same but somewhat similar to the case [7,8], we add terms breaking the exact supersymmetry to pick up a single vacuum corresponding to the desired continuum theory.

The classical actions of $\mathcal{N}=2 \mathrm{SYM}$ theories are written as the 'topological field theory (TFT) form': $Q$ (something). $Q$ is one of the supercharges of the SYM theories and nilpotent up to gauge transformations. Topological field theories are related to the $\mathcal{N}=2$ theories via the procedure of twist [12]. The twist changes the spin quantum numbers of fields, and some of supercharges become scalar. When regarding one of the scalar supercharges $Q$ as the BRST charge whose cohomology defines physical observables, the theories become topological field theories. In this paper, however we do not change the interpretation of the supercharge $Q$. Namely, $Q$ is regarded as just one of spinor supercharges of the original SYM theories not as the BRST operators. For theories on the flat space, the twisted theories are equivalent to the original theories, and the twist is solely renaming the field variables. We construct the lattice theories for $\mathcal{N}=2 \mathrm{SYM}$ theories in the dimensions $d=2,4,8$ keeping the exact supercharge $Q$. The lattice actions have the $Q$-exact form as well as the continuum ones. Among them, the $d=2$ case is shown to flow to the desired continuum theory without any fine tuning of parameters, where the supersymmetry breaking term does not alter the result of renormalization.

For $\mathcal{N}=4 \mathrm{SYM}$ theories, the actions are written as the form: $Q_{+} Q_{-}$(something), where $Q_{ \pm}$are two of the supercharges and nilpotent up to gauge transformations. We refer it as 'balanced topological field theory (BTFT) form'. Balanced topological field theories are also related to $\mathcal{N}=4 \mathrm{SYM}$ theories via the twist $[13,14,15]$. Similarly to the $\mathcal{N}=2$ case, we regard $Q_{ \pm}$as two of spinor supercharges of the $\mathcal{N}=4$ theories, and the twist amounts just to changing the notations of fields. The lattice models are constructed for $\mathcal{N}=4 \mathrm{SYM}$ in two- and four-dimensions preserving the $Q_{ \pm}$supersymmetries exactly. In this case, the lattice actions have $\mathrm{SU}(2)_{R}$ symmetry transforming $Q_{ \pm}$as a doublet, which is a subgroup of $\mathrm{SU}(4)$ internal symmetry of $\mathcal{N}=4$ theories. We show that the lattice theory for $\mathcal{N}=4$ in two-dimensions defines the corresponding continuum theory with no requirement for fine tuning as well as $\mathcal{N}=8$ theory in two-dimensions which is obtained by dimensional reduction from the $\mathcal{N}=4$ in four-dimensions.

This paper is organized as follows. In section 2, we briefly explain on the 'topological field theory form' of $\mathcal{N}=2 \mathrm{SYM}$ theories. In section 3 , we construct the lattice models for $\mathcal{N}=2$ theories in $d=2,4,8$, and show that the continuum theory is defined without fine tuning in the case $d=2$. In section 4 , we give some explanations on the 'balanced topological field theory form' for $\mathcal{N}=4$ SYM theories in two- and four-dimensions. In section 5 , we formulate the $\mathcal{N}=4$ theories on hypercubic lattices, and discuss on the renormalization in the continuum limit. Section 6 is devoted to summary and discussions. In appendix A, we give the explicit form of the $\gamma$-matrix conventions used in the paper. In appendix $\mathrm{B}$, we present the explicit form of the matrices $P_{\mu}$ for the $\mathcal{N}=2$ and 4 theories, which appear in the term analogous to the Wilson term removing the species doublers of fermions. In appendix $\mathrm{C}$, we discuss about $Q_{ \pm}$-cohomology in the $\mathcal{N}=4$ lattice models, whose result is useful for the renormalization argument in section 5 .

Throughout this paper, we focus on the gauge group $G=\mathrm{SU}(N)$. In sections 2 and 
4 discussing continuum theories, notations of repeated indices in formulas are assumed to be summed. On the other hand, in sections 3 and 5 for lattice theories, we explicitly write the summation over the indices except the cases of no possible confusion.

\section{2. 'Topological Field Theory Form' of $\mathcal{N}=2$ Super Yang-Mills Theories}

First, we give some explanation on Wick-rotated $\mathcal{N}=2$ SYM theories [16], and then move on their description in the 'topological field theory form'.

\subsection{Note on Wick-rotation and $\mathrm{U}(1)_{R}$ Symmetry}

We start with $\mathcal{N}=1 \mathrm{SYM}$ in $(D-1,1)$-dimensions $(D=4,6,10)$ :

$$
S^{(M)}=\frac{1}{g^{2}} \int \mathrm{d}^{D} x \operatorname{tr}\left[-\frac{1}{4} F^{M N} F_{M N}-\frac{i}{2} \bar{\Psi} \Gamma^{M} D_{M} \Psi\right],
$$

where $M, N=0,1, \cdots D-1$, and the metric tensor is $\eta^{M N}=\operatorname{diag}(-1,1,1, \cdots, 1)$. The $\Gamma$-matrices satisfy the Clifford algebra $\left\{\Gamma^{M}, \Gamma^{N}\right\}=-2 \eta^{M N}$. Here we take the Majorana representation for $\Gamma^{M}$. Namely, all of $\Gamma^{M}$ consist of pure imaginary entries. $\Gamma^{0}$ is antisymmetric, while $\Gamma^{i}(i=1, \cdots, D-1)$ are symmetric. The spinor field is expanded as $\Psi=\sum_{a} \Psi^{a} T^{a} . \Psi^{a}$ are real Grassmann valued spinors, and $T^{a}$ represent a basis of the gauge group generators. The number of spinor components is $2(D-2)$. Note

$$
\bar{\Psi}=\Psi^{T} \Gamma^{0}
$$

where the transpose acts only on spinor indices. The action is invariant under the Lorentz boost along the $x^{i}$ direction transforming the fermion as

$$
\Psi \rightarrow e^{\Gamma^{0} \Gamma^{i} \frac{\theta}{2}} \Psi
$$

After the Wick-rotation $\left(x^{0} \rightarrow-i x_{D}, \Gamma_{D} \equiv i \Gamma^{0}\right)$, the Euclidean action reads

$$
S^{(E)}=\frac{1}{g^{2}} \int \mathrm{d}^{D} x \operatorname{tr}\left[\frac{1}{4} F_{M N} F_{M N}+\frac{i}{2} \bar{\Psi} \Gamma_{M} D_{M} \Psi\right],
$$

with $\left\{\Gamma_{M}, \Gamma_{N}\right\}=-2 \delta_{M N}(M, N=1, \cdots, D)$. Now the Lorentz boost $(2.3)$ becomes a rotation in $(i, D)$-plane:

$$
\Psi \rightarrow e^{\Gamma_{D} \Gamma_{i} \frac{\theta}{2}} \Psi
$$

This rotation does not keep the property of $\Psi^{a}$ being real. Nevertheless, we can give a justification to regard the rotation as a symmetry of the action by still using (2.2) for $\bar{\Psi}$ in the action (2.4). We can interpret $\Psi^{a}$ as complexified Grassmann and the action as holomorphic with respect to $\Psi^{a}$ without changing the result of the path integral ${ }^{2}$. In the complexified space, (2.5) is closed and the rotation can be understood as a symmetry of the action.

\footnotetext{
${ }^{2}$ A complexified Grassmann number takes the form: (complex number) $\times$ (real Grassmann number).
} 
The action (2.4) is invariant under the supersymmetry transformation:

$$
\delta A_{M}=\epsilon^{T} \gamma_{M} \Psi, \quad \delta \Psi=-\frac{1}{2}\left(F_{i j} \gamma_{i j}+2 F_{i D} \gamma_{i}\right) \epsilon
$$

where $\epsilon$ is a real spinor parameter. Also, $\gamma_{i} \equiv-i \Gamma^{0} \Gamma_{i}, \gamma_{D} \equiv \mathbf{1}, \gamma_{i j}=\frac{1}{2}\left[\gamma_{i}, \gamma_{j}\right]$. Then, $\gamma_{i}$ satisfy $\left\{\gamma_{i}, \gamma_{j}\right\}=-2 \delta_{i j}$.

As a result of dimensional reduction with respect to the $x^{D-1}$ and $x^{D}$ directions, the theory (2.4) becomes $\mathcal{N}=2$ SYM theory in $d \equiv(D-2)$-dimensions. The rotational symmetry in $(D-1, D)$-plane is seen as $\mathrm{U}(1)_{R}$ internal symmetry in the $\mathcal{N}=2$ theory. Also, the $\mathcal{N}=2$ supersymmetry is given by the dimensional reduction of (2.6).

\section{2 'Topological Field Theory Form'}

The result of the dimensional reduction of the action (2.4) can be written as the 'topological field theory (TFT) form' $[12,17,18]$ :

$$
S_{\mathcal{N}=2}=Q \frac{1}{2 g_{d}^{2}} \int \mathrm{d}^{d} x \operatorname{tr}\left[\frac{1}{4} \eta[\phi, \bar{\phi}]-i \vec{\chi} \cdot \vec{\Phi}+\vec{\chi} \cdot \vec{H}-i \psi_{\mu} D_{\mu} \bar{\phi}\right]
$$

where $\mu$ is the index for $d$-dimensional space-time. Bosonic fields are $A_{\mu}, \phi \equiv A_{D-1}+i A_{D}$, $\bar{\phi} \equiv A_{D-1}-i A_{D}$, and the auxiliary fields $\vec{H}$. The other fields $\psi_{\mu}, \vec{\chi}, \eta$ are fermionic. $\vec{H}$ and $\vec{\chi}$ are column vectors with $(d-1)$-components. $\vec{\Phi}$ are functions of field strengths $F_{\mu \nu}$ and have one, three, seven components for the cases $d=2,4,8$, respectively. Their forms are given by

$$
\begin{array}{ll}
\Phi \equiv 2 F_{12} \quad \text { for } d=2 & \\
\Phi_{\mathrm{A}} \equiv 2\left(F_{\mathrm{A} 4}+\frac{1}{2} \varepsilon_{\mathrm{ABC}} F_{\mathrm{BC}}\right) & \text { for } d=4 \\
\Phi_{\mathrm{A}} \equiv 2\left(F_{\mathrm{A} 8}+\frac{1}{2} c_{\mathrm{ABC}} F_{\mathrm{BC}}\right) & \text { for } d=8 .
\end{array}
$$

$c_{\mathrm{ABC}}$ are totally antisymmetric and expressed as the structure constants of the algebra of octonions. Nonzero components are

$$
c_{127}=c_{163}=c_{154}=c_{253}=c_{246}=c_{347}=c_{567}=1
$$

and those obtained by index permutations therefrom.

$Q$ is one of the supercharges for the transformation (2.6), and its transformation rule is given as

$$
\begin{gathered}
Q A_{\mu}=\psi_{\mu}, \quad Q \psi_{\mu}=i D_{\mu} \phi \\
Q \phi=0, \\
Q \vec{\chi}=\vec{H}, \quad Q \vec{H}=[\phi, \vec{\chi}], \\
Q \bar{\phi}=\eta, \quad Q \eta=[\phi, \bar{\phi}] .
\end{gathered}
$$

$Q$ is nilpotent up to infinitesimal gauge transformations with the parameter $\phi$. By choosing the representation of $\gamma_{i}$ so that $\Psi$ takes the form

$$
\Psi^{T}=\left(\psi_{1}, \cdots, \psi_{d}, \chi_{1}, \cdots, \chi_{d-1}, \frac{1}{2} \eta\right)
$$


$Q$ is expressed as the transformation (2.6) with $\epsilon^{T}=(0, \cdots, 0,-\varepsilon) . \varepsilon$ is a real Grassmann number. See appendix A for the explicit form of $\gamma_{i}$. Since in the path integral we may regard $\phi$ and $\bar{\phi}$ as independent variables, it is not necessary to worry about the asymmetry with respect to $\phi$ and $\bar{\phi}$ in the transformation (2.12).

The action (2.7) is written more explicitly as

$$
\begin{aligned}
S_{\mathcal{N}=2}=\frac{1}{2 g_{d}^{2}} \int \mathrm{d}^{d} x \operatorname{tr} & {\left[\frac{1}{4}[\phi, \bar{\phi}]^{2}+\vec{H} \cdot \vec{H}-i \vec{H} \cdot \vec{\Phi}+D_{\mu} \phi D_{\mu} \bar{\phi}\right.} \\
& \left.-\frac{1}{4} \eta[\phi, \eta]-\vec{\chi} \cdot[\phi, \vec{\chi}]+\psi_{\mu}\left[\bar{\phi}, \psi_{\mu}\right]+i \vec{\chi} \cdot Q \vec{\Phi}+i \psi_{\mu} D_{\mu} \eta\right] .
\end{aligned}
$$

The charge for the $\mathrm{U}(1)_{R}$ rotation is assigned as follows: +2 for $\phi,-2$ for $\bar{\phi},+1$ for $\psi_{\mu}$, -1 for $\vec{\chi}$ and $\eta, 0$ for $A_{\mu}$ and $\vec{H}$. As is seen from the Yukawa interactions in (2.14), the $\mathrm{U}(1)_{R}$ is a chiral rotation which suffers anomaly generically in cases ${ }^{3} d=4,8$.

In refs. [12], topological field theory was obtained via the procedure of twist from theory with $\mathcal{N}=2$ supersymmetry. The twist changes the spin of fields and some supercharges become scalar. If one of the scalar supercharges is regarded as the BRST charge, whose cohomology and the gauge invariance determine physical observables, the theory becomes topological field theory. In the context of topological field theory, the $\mathrm{U}(1)_{R}$ charge stands for the ghost number, and the $\mathrm{U}(1)_{R}$ anomaly is seen as the formal dimension of instanton moduli space. The instanton is defined by configurations satisfying the equations $\vec{\Phi}=0$. Through the index theorem, the difference of the number of $\psi_{\mu}$ zero-modes (having the $\mathrm{U}(1)_{R}$ charge +1 ) and the number of $\vec{\chi}, \eta$ zero-modes (having the charge -1 ) gives the formal dimension of the instanton moduli space.

Note that in this paper we regard the theory (2.7) as $\mathcal{N}=2$ SYM theory itself, not as topological field theory. In fact, (2.7) is nothing but the $\mathcal{N}=2 \mathrm{SYM}$ action after renaming the fermionic fields as $(2.13)^{4}$ up to total derivative terms. Here, we consider $Q$ as one of the spinor supercharges and do not use it to define physical observables. Physical operators are determined solely by gauge invariance of the $\mathcal{N}=2$ SYM theory. From this view-point, the topological field theory is considered as a special subsector of the original $\mathcal{N}=2$ supersymmetric theory.

The total derivative terms exist in the 'TFT form' (2.7) for the cases $d=4,8$. They are explicitly written as

$$
\begin{aligned}
& \frac{1}{2 g_{4}^{2}} \int \operatorname{tr}(F \wedge F) \quad \text { for } d=4, \\
& \frac{1}{2 g_{8}^{2}} \int \Omega \wedge \operatorname{tr}(F \wedge F) \quad \text { for } d=8,
\end{aligned}
$$

where $F=\frac{1}{2} F_{\mu \nu} \mathrm{d} x^{\mu} \wedge \mathrm{d} x^{\nu}$, and $\Omega$ is a self-dual closed four-form:

$$
\begin{aligned}
& \Omega=\omega+* \omega \\
& \omega=\frac{1}{3 !} c_{\mathrm{ABC}} \mathrm{d} x^{\mathrm{A}} \wedge \mathrm{d} x^{\mathrm{B}} \wedge \mathrm{d} x^{\mathrm{C}} \wedge \mathrm{d} x^{8} .
\end{aligned}
$$

\footnotetext{
${ }^{3}$ In two-dimensions, since the Pontryagin class is $\operatorname{tr} F$, it vanishes for $G=\mathrm{SU}(N)$ and the chiral symmetry $\mathrm{U}(1)_{R}$ is not anomalous.

${ }^{4}$ Note that the index $\mu$ of $\psi_{\mu}$ does not reflect the Lorentz transformation property.
} 


\section{Lattice Formulation with One Exact Supercharge}

In this section, we formulate the theory (2.7) on the $d$-dimensional hypercubic lattice keeping the supersymmetry $Q$. In the lattice theory, gauge fields $A_{\mu}(x)$ are promoted to the compact unitary variables

$$
U_{\mu}(x)=e^{i a A_{\mu}(x)}
$$

on the link $(x, x+\hat{\mu})$. ' $a$ ' stands for the lattice spacing, and $x \in \mathbf{Z}^{d}$ the lattice site. All other variables are distributed at sites. Interestingly, the $Q$-transformation (2.12) is extendible to the lattice variables preserving the property

$$
Q^{2}=\text { (infinitesimal gauge transformation with the parameter } \phi \text { ) }
$$

as follows:

$$
\begin{aligned}
& Q U_{\mu}(x)=i \psi_{\mu}(x) U_{\mu}(x), \\
& Q \psi_{\mu}(x)=i \psi_{\mu}(x) \psi_{\mu}(x)-i\left(\phi(x)-U_{\mu}(x) \phi(x+\hat{\mu}) U_{\mu}(x)^{\dagger}\right), \\
& Q \phi(x)=0, \\
& Q \vec{\chi}(x)=\vec{H}(x), \quad Q \vec{H}(x)=[\phi(x), \vec{\chi}(x)], \\
& Q \bar{\phi}(x)=\eta(x), \quad Q \eta(x)=[\phi(x), \bar{\phi}(x)] .
\end{aligned}
$$

Also, $Q U_{\mu}(x)^{\dagger}=-i U_{\mu}(x)^{\dagger} \psi_{\mu}(x)$ follows from $U_{\mu}(x) U_{\mu}(x)^{\dagger}=1$. All transformations except $Q U_{\mu}(x)$ and $Q \psi_{\mu}(x)$ are of the same form as in the continuum case. Since (3.2) means

$$
\begin{aligned}
Q^{2} U_{\mu}(x) & =i\left(Q \psi_{\mu}(x)\right) U_{\mu}(x)-i \psi_{\mu}(x)\left(Q U_{\mu}(x)\right) \\
& =\phi(x) U_{\mu}(x)-U_{\mu}(x) \phi(x+\hat{\mu}),
\end{aligned}
$$

if we assume the formula " $Q U_{\mu}(x)=\cdots$ ", the transformation $Q \psi_{\mu}(x)$ is determined" . Then, happily $Q^{2} \psi_{\mu}(x)=\left[\phi(x), \psi_{\mu}(x)\right]$ is satisfied, and the $Q$-transformation is consistently closed. Note that we use the dimensionless variables here, and that various quantities are of the following orders:

$$
\begin{aligned}
& \psi_{\mu}(x), \vec{\chi}(x), \eta(x)=O\left(a^{3 / 2}\right), \quad \phi(x), \bar{\phi}(x)=O(a), \quad \vec{H}(x)=O\left(a^{2}\right), \\
& Q=O\left(a^{1 / 2}\right) .
\end{aligned}
$$

The first term in the RHS of " $Q \psi_{\mu}(x)=\ldots$ " in (3.3) is of subleading order $O\left(a^{3}\right)$.

\subsection{Lattice Action}

Once we have the $Q$-transformation rule closed among lattice variables, it is almost straightforward to construct the lattice action with the exact supersymmetry $Q$ :

$$
\begin{aligned}
S_{\mathcal{N}=2}^{\mathrm{LAT}}=Q \frac{1}{2 g_{0}^{2}} \sum_{x} \operatorname{tr} & {\left[\frac{1}{4} \eta(x)[\phi(x), \bar{\phi}(x)]-i \vec{\chi}(x) \cdot \vec{\Phi}(x)+\vec{\chi}(x) \cdot \vec{H}(x)\right.} \\
& \left.+i \sum_{\mu=1}^{d} \psi_{\mu}(x)\left(\bar{\phi}(x)-U_{\mu}(x) \bar{\phi}(x+\hat{\mu}) U_{\mu}(x)^{\dagger}\right)\right],
\end{aligned}
$$

\footnotetext{
${ }^{5}$ The first term in the RHS does not vanish because $i \psi_{\mu}(x) \psi_{\mu}(x)=-\frac{1}{2} f^{a b c} \psi_{\mu}^{a}(x) \psi_{\mu}^{b}(x) T^{c}$ with $f^{a b c}$ being structure constants of the gauge group.
} 
where

$$
\begin{aligned}
& \Phi(x)=-i\left[U_{12}(x)-U_{21}(x)\right] \quad \text { for } d=2, \\
& \Phi_{\mathrm{A}}(x)=-i\left[U_{\mathrm{A} 4}(x)-U_{4 \mathrm{~A}}(x)+\frac{1}{2} \sum_{\mathrm{B}, \mathrm{C}=1}^{3} \varepsilon_{\mathrm{ABC}}\left(U_{\mathrm{BC}}(x)-U_{\mathrm{CB}}(x)\right)\right] \quad \text { for } d=4, \\
& \Phi_{\mathrm{A}}(x)=-i\left[U_{\mathrm{A} 8}(x)-U_{8 \mathrm{~A}}(x)+\frac{1}{2} \sum_{\mathrm{B}, \mathrm{C}=1}^{7} c_{\mathrm{ABC}}\left(U_{\mathrm{BC}}(x)-U_{\mathrm{CB}}(x)\right)\right] \quad \text { for } d=8 .
\end{aligned}
$$

$U_{\mu \nu}(x)$ are plaquette variables written as

$$
U_{\mu \nu}(x) \equiv U_{\mu}(x) U_{\nu}(x+\hat{\mu}) U_{\mu}(x+\hat{\nu})^{\dagger} U_{\nu}(x)^{\dagger} .
$$

The action (3.6) is clearly $Q$-invariant from its $Q$-exact form. After acting $Q$ in the RHS, the action takes the form

$$
\begin{aligned}
S_{\mathcal{N}=2}^{\mathrm{LAT}}=\frac{1}{2 g_{0}^{2}} \sum_{x} \operatorname{tr} & {\left[\frac{1}{4}[\phi(x), \bar{\phi}(x)]^{2}+\vec{H}(x) \cdot \vec{H}(x)-i \vec{H}(x) \cdot \vec{\Phi}(x)\right.} \\
& +\sum_{\mu=1}^{d}\left(\phi(x)-U_{\mu}(x) \phi(x+\hat{\mu}) U_{\mu}(x)^{\dagger}\right)\left(\bar{\phi}(x)-U_{\mu}(x) \bar{\phi}(x+\hat{\mu}) U_{\mu}(x)^{\dagger}\right) \\
& -\frac{1}{4} \eta(x)[\phi(x), \eta(x)]-\vec{\chi}(x) \cdot[\phi(x), \vec{\chi}(x)] \\
& -\sum_{\mu=1}^{d} \psi_{\mu}(x) \psi_{\mu}(x)\left(\bar{\phi}(x)+U_{\mu}(x) \bar{\phi}(x+\hat{\mu}) U_{\mu}(x)^{\dagger}\right) \\
& \left.\left.+i \vec{\chi}(x) \cdot Q \vec{\Phi}(x)-i \sum_{\mu=1}^{d} \psi_{\mu}(x)\left(\eta(x)-U_{\mu}(x) \eta(x+\hat{\mu}) U_{\mu}(x)^{\dagger}\right)\right] .(3.11)\right]
\end{aligned}
$$

After integrating out $\vec{H}(x)$, induced $\vec{\Phi}(x)^{2}$ term yields the gauge kinetic term as the form

$$
\frac{1}{2 g_{0}^{2}} \sum_{x} \sum_{\mu<\nu} \operatorname{tr}\left[-\left(U_{\mu \nu}(x)-U_{\nu \mu}(x)\right)^{2}\right]
$$

which leads double winding plaquette terms. Note difference from the standard Wilson action

$$
\frac{1}{2 g_{0}^{2}} \sum_{x} \sum_{\mu<\nu} \operatorname{tr}\left[2-U_{\mu \nu}(x)-U_{\nu \mu}(x)\right] .
$$

Also, for $d=4,8$, there appear terms corresponding to the total derivatives (2.15).

In contrast with (3.13) giving the unique minimum $U_{\mu \nu}(x)=1$, the action (3.12) has many classical vacua

$$
U_{\mu \nu}(x)=\left(\begin{array}{lll} 
\pm 1 & & \\
& \ddots & \\
& & \pm 1
\end{array}\right)
$$


up to gauge transformations, where any combinations of \pm 1 with ' -1 ' appearing even times are allowed in the diagonal entries. The number of ' -1 ' means strength of a color flux through the plaquette. Since the configurations (3.14) can be taken freely for each plaquette, it leads a huge degeneracy of vacua with the number growing as exponential of the number of plaquettes. In order to see the dynamics of the model, we need to sum up contributions from all the minima, and the ordinary weak field expansion around a single vacuum $U_{\mu \nu}(x)=1$ can not be justified ${ }^{6}$. Thus, we can not say anything on the continuum limit of the lattice model (3.11) without its nonperturbative investigations.

Aiming to resolve the degeneracy, we add the following term to the action:

$$
\Delta S=\frac{1}{2 g_{0}^{2}} \rho \sum_{x} \sum_{\mu<\nu} \operatorname{tr}\left(2-U_{\mu \nu}(x)-U_{\nu \mu}(x)\right)
$$

which is proportional to the standard Wilson action (3.13), resolving the degeneracy with the split $\frac{4 \rho}{g_{0}^{2}}$. We will take the coefficient $\rho$ so that $\Delta S$ vanishes in the classical continuum limit but then the split grows infinite. For example, in case of the two-dimensional $M \times M$ periodic lattice, the continuum limit is taken as $a \rightarrow 0$ with $g_{2}^{2} \equiv g_{0}^{2} a^{-2}$ and $L \equiv M a$ fixed. $\rho$ can be chosen as $\rho=\frac{1}{M^{s}}=\frac{a^{s}}{L^{s}}$ with the parameter $0<s<2$. Since the number of the lifted vacua with $\Delta S=\frac{4 \rho}{g_{0}^{2}}=\frac{4}{g_{2}^{2} L^{s}} \frac{1}{a^{2-s}}$ is proportional to the number of plaquettes, the entropy effect does not obstruct the decoupling. While this term justifies the expansion around $U_{\mu \nu}(x)=1$, it breaks the supersymmetry $Q$. As is seen in section 3.3 , however in the case of $d=2$ it does not affect the renormalization argument.

The last two terms in (3.11) give rise to the fermion kinetic term. In particular, $\vec{\chi}(x) \cdot Q \vec{\Phi}(x)$ has the plaquette structure where two fermions sit at the same site or separately at the nearest neighbor sites. As an example, Fig. 1 shows the situation for $d=2$ case. Owing to this structure, the lattice model does not have 90-degree rotational symmetry or the reflection positivity, either. However for $d=2$ case the model is shown to flow to the desired continuum theory without any fine tuning of parameters.

\subsection{Absence of Fermion Doubling}

We expand the exponential of the link variable (3.1), and look at the kinetic terms in the action (3.11). Because in bosonic sector no species doublers appear, in fermionic sector also no doublers are expected due to the exact supersymmetry $Q$ of (3.11). Let us see the fermionic sector explicitly.

After rescaling each fermion variable by $a^{3 / 2}$ as indicated in (3.5), the fermion kinetic terms are expressed as

$$
\begin{aligned}
S_{f}^{(2)} & =\frac{a^{4}}{2 g_{0}^{2}} \sum_{x, \mu} \operatorname{tr}\left[-\frac{1}{2} \Psi(x)^{T}\left(P_{\mu}+\gamma_{\mu}\right) \Delta_{\mu} \Psi(x)+\frac{1}{2} \Psi(x)^{T}\left(P_{\mu}-\gamma_{\mu}\right) \Delta_{\mu}^{*} \Psi(x)\right] \\
& =\frac{a^{4}}{2 g_{0}^{2}} \sum_{x, \mu} \operatorname{tr}\left[-\frac{1}{2} \Psi(x)^{T} \gamma_{\mu}\left(\Delta_{\mu}+\Delta_{\mu}^{*}\right) \Psi(x)-a \frac{1}{2} \Psi(x)^{T} P_{\mu} \Delta_{\mu} \Delta_{\mu}^{*} \Psi(x)\right]
\end{aligned}
$$

\footnotetext{
${ }^{6}$ This is the same difficulty already encountered in ref. [4]. We thank Y. Shamir for the crucial comment.
} 


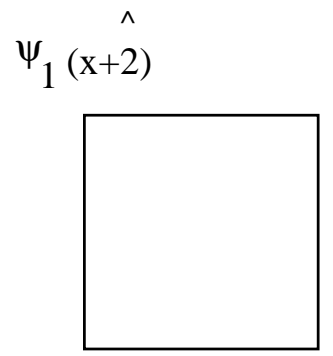

$\chi(\mathrm{x})$

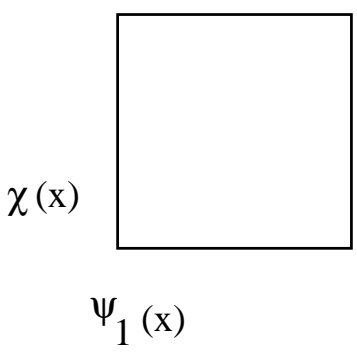

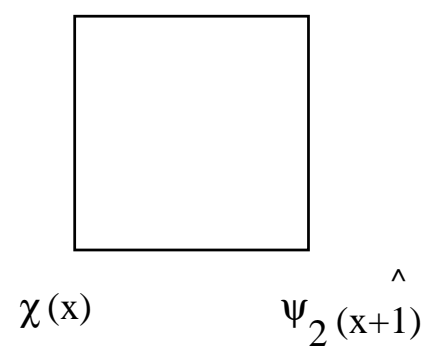

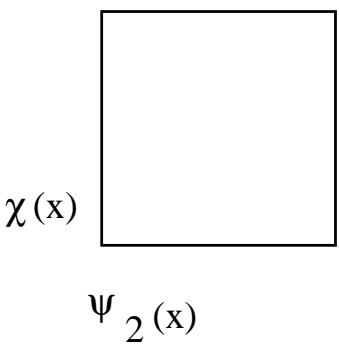

Figure 1: The plaquette structure of the term $\vec{\chi}(x) \cdot Q \vec{\Phi}(x)$ for $d=2$ case. The variable $U_{\mu}$ is assigned on each link. Fermions $\chi$ and $\psi_{\mu}$ are distributed in the depicted four patterns.

where fermions were combined as (2.13). $P_{\mu}$ are hermitian matrices satisfying $\left\{P_{\mu}, P_{\nu}\right\}=$ $2 \delta_{\mu \nu}$. For their explicit form, see appendix B. $\Delta_{\mu}$ and $\Delta_{\mu}^{*}$ represent forward and backward difference operators respectively:

$$
\Delta_{\mu} f(x) \equiv \frac{1}{a}(f(x+\hat{\mu})-f(x)), \quad \Delta_{\mu}^{*} f(x) \equiv \frac{1}{a}(f(x)-f(x-\hat{\mu})) .
$$

The last term containing $P_{\mu}$ has a similar structure to the Wilson term, and plays a role of removing fermion doublers. The lattice action is $\mathrm{U}(1)_{R}$ invariant, and the fermion doublers are removed keeping the chiral $\mathrm{U}(1)_{R}$. Thus, the model must break some assumptions of Nielsen-Ninomiya's no go theorem [19]. In fact, broken is the assumption "There exists a conserved charge $Q_{F}$ corresponding to the fermion number.". For simplicity, let us see the situation in $d=2$ case. When combining fermions into a two-component Dirac spinor as

$$
\zeta=\frac{1}{\sqrt{2}}\left(\begin{array}{c}
\psi_{1}-i \psi_{2} \\
\chi+i \frac{1}{2} \eta
\end{array}\right), \quad \bar{\zeta}=\frac{1}{\sqrt{2}}\left(\psi_{1}+i \psi_{2}, \chi-i \frac{1}{2} \eta\right)
$$

$Q_{F}$ corresponds to the following $\mathrm{U}(1)_{J}$ rotation:

$$
\zeta \rightarrow e^{i \theta} \zeta, \quad \bar{\zeta} \rightarrow e^{-i \theta} \bar{\zeta}
$$

The first term in (3.16), giving a naive fermion kinetic term on the lattice, is written as the combination $\bar{\zeta}_{\alpha} \zeta_{\beta}$, which is invariant under the $\mathrm{U}(1)_{J}$. On the other hand, the last term 


\begin{tabular}{|c|c|c|c|c|c|}
\hline $\bar{p} p=a+b+3 c$ & \multicolumn{5}{|c|}{$\overline{\varphi^{a} \partial^{b} \psi^{2 c}}$} \\
\hline 0 & \multicolumn{5}{|c|}{1} \\
\hline 1 & \multicolumn{5}{|c|}{$\varphi$} \\
\hline 2 & & & $\varphi^{2}$ & & \\
\hline 3 & & $\varphi^{3}$, & $\psi \psi$ & $\varphi \partial \varphi$ & \\
\hline 4 & $\varphi^{4}$, & $\varphi^{2} \partial \varphi$ & $(\partial \varphi)^{2}$ & $\psi \partial \psi$ & $\varphi \psi \psi$ \\
\hline
\end{tabular}

Table 1: List of operators with $p \leq 4$.

containing $P_{\mu}$ takes the form:

$$
\frac{a^{4}}{2 g_{0}^{2}} \sum_{x} \frac{a}{2} \operatorname{tr}\left[\varepsilon_{\alpha \beta} \zeta_{\alpha}\left(\Delta_{1} \Delta_{1}^{*}+i \Delta_{2} \Delta_{2}^{*}\right) \zeta_{\beta}-\varepsilon_{\alpha \beta} \bar{\zeta}_{\alpha}\left(\Delta_{1} \Delta_{1}^{*}-i \Delta_{2} \Delta_{2}^{*}\right) \bar{\zeta}_{\beta}\right]
$$

to break the $\mathrm{U}(1)_{J}$ invariance.

\subsection{Renormalization}

At the classical level, the lattice action (3.6) leads to the continuum action (2.7) in the limit $a \rightarrow 0$ with $g_{d}^{-2} \equiv a^{4-d} g_{0}^{-2}$ kept fixed, and thus the $\mathcal{N}=2$ supersymmetry and rotational symmetry in $d$-dimensions are restored. We will check whether the symmetry restoration persists against quantum corrections, i.e. whether symmetry of the lattice action forbids any relevant or marginal operators induced which obstruct the symmetry restoration.

Here, we consider the case $d=2$ only, where the $\mathrm{U}(1)_{R}$ symmetry is not anomalous. Since the model has the critical point $g_{0}=0$ from the asymptotic freedom, we shall consider the renormalization effect perturbatively. For a while, we discuss without the supersymmetry breaking term $\Delta S$. The mass dimension of the coupling $g_{2}^{2}$ is two. For generic boson field $\varphi$ (other than the auxiliary fields) and fermion field $\psi$, the dimensions are 1 and $3 / 2$ respectively. Thus, operators of the type $\varphi^{a} \partial^{b} \psi^{2 c}$ have the dimension $p \equiv$ $a+b+3 c$, where ' $\partial$ ' means a derivative with respect to the coordinates. From dimensional analysis, the operators receive the following radiative corrections up to some powers of possible logarithmic factors:

$$
\left(\frac{a^{p-4}}{g_{2}^{2}}+c_{1} a^{p-2}+c_{2} a^{p} g_{2}^{2}+\cdots\right) \int \mathrm{d}^{2} x \varphi^{a} \partial^{b} \psi^{2 c},
$$

where $c_{1}, c_{2}, \cdots$ are constants dependent on $N$. The first, second and third terms in the parentheses represent the contributions at tree, one-loop and two-loop levels. It is easily seen from the fact that $g_{2}^{2}$ appears as an overall factor in front of the action and plays the same role as the Planck constant $\hbar$. Due to the super-renormalizable property in twodimensional theory, the relevant corrections terminate at the two-loop. From the above formula, it is seen that the following operators can be relevant or marginal in the $a \rightarrow 0$ limit: operators with $p \leq 2$ induced at the one-loop level and with $p=0$ at the two-loop level. Operators with $p \leq 4$ are listed in Table 1 .

Since the identity operator does not affect the spectrum, we have to check operators of the types $\varphi$ and $\varphi^{2}$ only. Gauge symmetry and $\mathrm{U}(1)_{R}$ invariance allow the operator $\operatorname{tr} \phi \bar{\phi}$, 
while it is forbidden by the supersymmetry $Q$. Hence, no relevant or marginal operators except the identity are generated by radiative corrections.

When taking into account the term $\Delta S$, we still have gauge invariance and $\mathrm{U}(1)_{R}$ symmetry but not the supersymmetry $Q$. This time, $\operatorname{tr} \phi \bar{\phi}$ might seem to remain. However, such is not the case. The supersymmetry breaking effect comes from loop diagrams containing the vertices of $\Delta S$, and insertion of $n \Delta S$-vertices yields the extra factor $\rho^{n}=\frac{a^{n s}}{L^{n s}}$ to each term in (3.21). So we can see that contribution from the supersymmetry breaking term to $\operatorname{tr} \phi \bar{\phi}$ becomes irrelevant behaving as $a^{n s}$. The conclusion of the renormalization argument without $\Delta S$ is not affected by including the breaking term. In the continuum limit, thus full supersymmetry and rotational symmetry are considered to be restored without any fine tuning.

\section{4. 'Balanced Topological Field Theory Form' of $\mathcal{N}=4$ Super Yang-Mills Theories}

In this section, we consider $\mathcal{N}=4 \mathrm{SYM}$ theories in two- and four-dimensions, which are obtained by further dimensional reduction from the $\mathcal{N}=2$ theories in $d=4,8$, respectively. The theories can be written as exact forms with respect to both of two supercharges, which we refer as 'balanced topological field theory (BTFT) form' $[13,14,15]$. As the name 'balanced' means, fields carrying the positive $\mathrm{U}(1)_{R}$ charge are balanced with those carrying the negative charge, and thus the $\mathrm{U}(1)_{R}$ symmetry is not anomalous.

\subsection{Two-dimensional Case}

After dimensional reduction with respect to the $x^{3}$ and $x^{4}$ directions, the $\mathcal{N}=2 \mathrm{SYM}$

in $d=4$ becomes two-dimensional $\mathcal{N}=4$ SYM theory, which is written as the following 'BTFT form':

$$
\begin{aligned}
& S_{\mathcal{N}=4}^{2 d}=Q_{+} Q_{-} \mathcal{F}_{\mathcal{N}=4}^{2 d}, \\
& \mathcal{F}_{\mathcal{N}=4}^{2 d}=\frac{1}{2 g_{2}^{2}} \int \mathrm{d}^{2} x \operatorname{tr}\left[-i B \Phi-\psi_{+\mu} \psi_{-\mu}-\chi_{+} \chi_{-}-\frac{1}{4} \eta_{+} \eta_{-}\right]
\end{aligned}
$$

where $Q_{ \pm}$are two of supercharges of the $\mathcal{N}=4$ theory specified below, and $\Phi \equiv 2 F_{12}$. Bosons are gauge fields $A_{\mu}(\mu=1,2)$ and scalar fields $B \equiv A_{3}, C \equiv 2 A_{4}, \phi, \bar{\phi}$. Also, there are auxiliary fields $\tilde{H}_{\mu}, H$. Scalars $B$ and $C$ appear in the process of the dimensional reduction. Other fields $\psi_{ \pm \mu}, \chi_{ \pm}, \eta_{ \pm}$are fermions. Transformation rule of the supersymmetry $Q_{ \pm}$is given by

$$
\begin{aligned}
Q_{+} A_{\mu} & =\psi_{+\mu}, \quad Q_{+} \psi_{+\mu}=i D_{\mu} \phi, \quad Q_{-} \psi_{+\mu}=\frac{i}{2} D_{\mu} C-\tilde{H}_{\mu}, \\
Q_{-} A_{\mu} & =\psi_{-\mu}, \quad Q_{-} \psi_{-\mu}=-i D_{\mu} \bar{\phi}, \quad Q_{+} \psi_{-\mu}=\frac{i}{2} D_{\mu} C+\tilde{H}_{\mu}, \\
Q_{+} \tilde{H}_{\mu} & =\left[\phi, \psi_{-\mu}\right]-\frac{1}{2}\left[C, \psi_{+\mu}\right]-\frac{i}{2} D_{\mu} \eta_{+}, \\
Q_{-} \tilde{H}_{\mu} & =\left[\bar{\phi}, \psi_{+\mu}\right]+\frac{1}{2}\left[C, \psi_{-\mu}\right]+\frac{i}{2} D_{\mu} \eta_{-},
\end{aligned}
$$




$$
\begin{aligned}
& Q_{+} B=\chi_{+}, \quad Q_{+} \chi_{+}=[\phi, B], \quad Q_{-} \chi_{+}=\frac{1}{2}[C, B]-H, \\
& Q_{-} B=\chi_{-}, \quad Q_{-} \chi_{-}=-[\bar{\phi}, B], \quad Q_{+} \chi_{-}=\frac{1}{2}[C, B]+H, \\
& Q_{+} H=\left[\phi, \chi_{-}\right]+\frac{1}{2}\left[B, \eta_{+}\right]-\frac{1}{2}\left[C, \chi_{+}\right], \\
& Q_{-} H=\left[\bar{\phi}, \chi_{+}\right]-\frac{1}{2}\left[B, \eta_{-}\right]+\frac{1}{2}\left[C, \chi_{-}\right], \\
& Q_{+} C=\eta_{+}, \quad Q_{+} \eta_{+}=[\phi, C], \quad Q_{-} \eta_{+}=-[\phi, \bar{\phi}], \\
& Q_{-} C=\eta_{-}, \quad Q_{-} \eta_{-}=-[\bar{\phi}, C], \quad Q_{+} \eta_{-}=[\phi, \bar{\phi}], \\
& Q_{+} \phi=0, \quad Q_{-} \phi=-\eta_{+}, \quad Q_{+} \bar{\phi}=\eta_{-}, \quad Q_{-} \bar{\phi}=0 .
\end{aligned}
$$

The transformation leads the following nilpotency of $Q_{ \pm}$(up to gauge transformations):

$$
\begin{aligned}
Q_{+}^{2} & =(\text { infinitesimal gauge transformation with the parameter } \phi), \\
Q_{-}^{2} & =(\text { infinitesimal gauge transformation with the parameter }-\bar{\phi}), \\
\left\{Q_{+}, Q_{-}\right\} & =(\text {infinitesimal gauge transformation with the parameter } C) .
\end{aligned}
$$

Since $\mathcal{F}_{\mathcal{N}=4}^{2 d}$ is gauge invariant, clearly $S_{\mathcal{N}=4}^{2 d}$ is invariant under the $Q_{ \pm}$transformations. Note that the form of $\mathcal{F}_{\mathcal{N}=4}^{2 d}$ is not unique because the action is invariant under the shift

$$
\mathcal{F}_{\mathcal{N}=4}^{2 d} \rightarrow \mathcal{F}_{\mathcal{N}=4}^{2 d}+Q_{+} G_{-}+Q_{-} G_{+}
$$

with gauge invariant $G_{ \pm}$.

With the same $\gamma$-matrix convention as the $d=4$ case in appendix A, notations for fermions in the 'TFT form' (2.13) correspond to

$$
\Psi^{T}=\left(\psi_{+1}, \psi_{+2}, \chi_{+}, \frac{1}{2} \eta_{+}, \psi_{-1}, \psi_{-2}, \chi_{-}, \frac{1}{2} \eta_{-}\right)
$$

$Q_{+}$is nothing but the dimensional reduction of $Q$ of the $\mathcal{N}=2, d=4$ case, and $Q_{-}$ represents the transformation (2.6) with $\epsilon^{T}=(0,0,0,-\varepsilon, 0,0,0,0)$. $\varepsilon$ is real Grassmann.

Under the dimensional reduction, the $\mathrm{U}(1)_{R}$ internal symmetry enhances to $\mathrm{SU}(2)_{R}$, which is a subgroup of $\mathrm{SU}(4)$ internal symmetry of $\mathcal{N}=4$ theory. The $\mathrm{SU}(2)_{R}$ generators are expressed as

$$
\begin{aligned}
J_{++}=\int \mathrm{d}^{2} x & {\left[\psi_{+\mu}^{a}(x) \frac{\delta}{\delta \psi_{-\mu}^{a}(x)}+\chi_{+}^{a}(x) \frac{\delta}{\delta \chi_{-}^{a}(x)}-\eta_{+}^{a}(x) \frac{\delta}{\delta \eta_{-}^{a}(x)}+2 \phi^{a}(x) \frac{\delta}{\delta C^{a}(x)}\right.} \\
- & \left.C^{a}(x) \frac{\delta}{\delta \bar{\phi}^{a}(x)}\right], \\
J_{--}=\int \mathrm{d}^{2} x & {\left[\psi_{-\mu}^{a}(x) \frac{\delta}{\delta \psi_{+\mu}^{a}(x)}+\chi_{-}^{a}(x) \frac{\delta}{\delta \chi_{+}^{a}(x)}-\eta_{-}^{a}(x) \frac{\delta}{\delta \eta_{+}^{a}(x)}-2 \bar{\phi}^{a}(x) \frac{\delta}{\delta C^{a}(x)}\right.} \\
+ & \left.C^{a}(x) \frac{\delta}{\delta \phi^{a}(x)}\right],
\end{aligned}
$$




$$
\begin{aligned}
J_{0}=\int \mathrm{d}^{2} x & {\left[\psi_{+\mu}^{a}(x) \frac{\delta}{\delta \psi_{+\mu}^{a}(x)}-\psi_{-\mu}^{a}(x) \frac{\delta}{\delta \psi_{-\mu}^{a}(x)}+\chi_{+}^{a}(x) \frac{\delta}{\delta \chi_{+}^{a}(x)}-\chi_{-}^{a}(x) \frac{\delta}{\delta \chi_{-}^{a}(x)}\right.} \\
+ & \left.\eta_{+}^{a}(x) \frac{\delta}{\delta \eta_{+}^{a}(x)}-\eta_{-}^{a}(x) \frac{\delta}{\delta \eta_{-}^{a}(x)}+2 \phi^{a}(x) \frac{\delta}{\delta \phi^{a}(x)}-2 \bar{\phi}^{a}(x) \frac{\delta}{\delta \bar{\phi}^{a}(x)}\right],
\end{aligned}
$$

with ' $a$ ' being the index of a basis of the gauge group generators, and satisfy the algebra:

$$
\left[J_{0}, J_{++}\right]=2 J_{++}, \quad\left[J_{0}, J_{--}\right]=-2 J_{--}, \quad\left[J_{++}, J_{--}\right]=J_{0} .
$$

$J_{0}$ is a generator of the $\mathrm{U}(1)_{R}$ rotation, which is contained in $\mathrm{SU}(2)_{R}$ as its Cartan subalgebra. $J_{++}\left(J_{--}\right)$raises (lowers) the $\mathrm{U}(1)_{R}$ charge by two-units. For fermions, subscript \pm means the $\mathrm{U}(1)_{R}$ charge \pm 1 , and $Q_{ \pm}$raises/lowers the charge by one. For auxiliary fields $\tilde{H}_{\mu}, H$, the charge zero is assigned. Under the $\mathrm{SU}(2)_{R}$, each of $\left(\psi_{+\mu}^{a}, \psi_{-\mu}^{a}\right),\left(\chi_{+}^{a}, \chi_{-}^{a}\right)$, $\left(\eta_{+}^{a},-\eta_{-}^{a}\right)$ and $\left(Q_{+}, Q_{-}\right)$transforms as a doublet, and $\left(\phi^{a}, C^{a},-\bar{\phi}^{a}\right)$ as a triplet.

The $Q_{ \pm}$-transformation properties of (4.2), (4.3) and (4.4) are symbolically summarized as
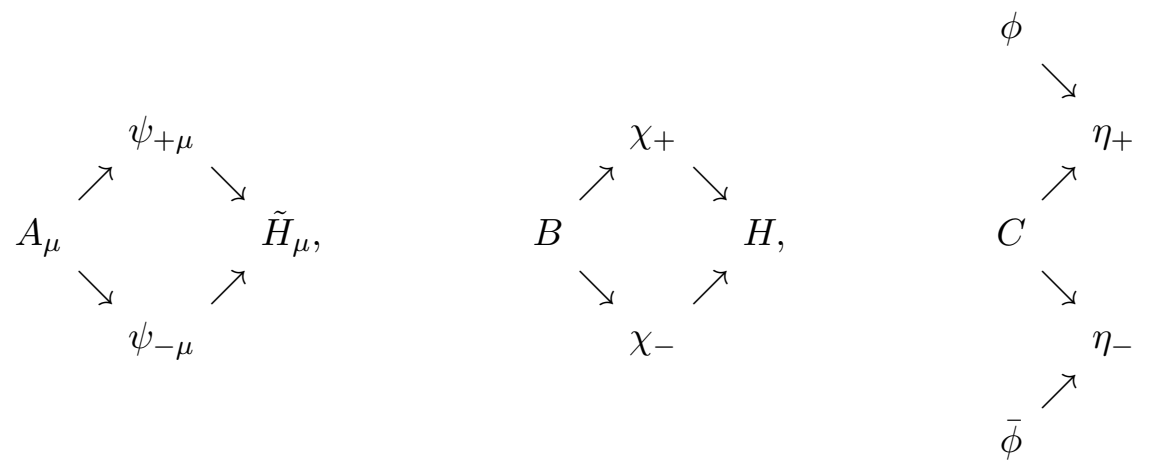

where $\nearrow$ and $\searrow$ represent the actions of $Q_{+}$and $Q_{-}$respectively. The vertical direction indicates the $\mathrm{U}(1)_{R}$ charge (From the top, $+2,+1,0,-1,-2$.).

Finally, let us note a symmetry of the action (4.1) under exchanging the two supercharges $Q_{+} \leftrightarrow Q_{-}$with

$$
\begin{aligned}
& \phi \rightarrow-\bar{\phi}, \quad \bar{\phi} \rightarrow-\phi, \quad B \rightarrow-B, \\
& \chi_{+} \rightarrow-\chi_{-}, \quad \chi_{-} \rightarrow-\chi_{+}, \quad \tilde{H}_{\mu} \rightarrow-\tilde{H}_{\mu}, \\
& \psi_{ \pm \mu} \rightarrow \psi_{\mp \mu}, \quad \eta_{ \pm} \rightarrow \eta_{\mp} .
\end{aligned}
$$

\subsection{Four-dimensional Case}

Dimensional reduction from the $\mathcal{N}=2$ theory in $d=8$ with respect to the $x^{5}, \cdots, x^{8}$ directions leads four-dimensional $\mathcal{N}=4$ SYM theory. Its 'BTFT form' is as follows:

$$
\begin{aligned}
& S_{\mathcal{N}=4}^{4 d}=Q_{+} Q_{-} \mathcal{F}_{\mathcal{N}=4}^{4 d}, \\
& \mathcal{F}_{\mathcal{N}=4}^{4 d}=\frac{1}{2 g_{4}^{2}} \int \mathrm{d}^{4} x \operatorname{tr}\left[-i \vec{B} \cdot \vec{\Phi}-\frac{1}{3} \varepsilon_{\mathrm{ABC}} B_{\mathrm{A}}\left[B_{\mathrm{B}}, B_{\mathrm{C}}\right]\right. \\
& \left.\quad-\psi_{+\mu} \psi_{-\mu}-\vec{\chi}_{+} \cdot \vec{\chi}_{-}-\frac{1}{4} \eta_{+} \eta_{-}\right]
\end{aligned}
$$


where $\mu=1, \cdots, 4$, and $\vec{\Phi}, \vec{B}, \vec{\chi}_{ \pm}$are three-component vectors with the indices A, B, C = $1,2,3 . \Phi_{\mathrm{A}}$ are functions of the field strengths $F_{\mu \nu}: \Phi_{\mathrm{A}}=2\left(F_{\mathrm{A} 4}+\frac{1}{2} \varepsilon_{\mathrm{ABC}} F_{\mathrm{BC}}\right)$, Bosonic field contents are gauge fields $A_{\mu}(\mu=1, \cdots, 4)$ and scalar fields $B_{1} \equiv-A_{5}, B_{2} \equiv A_{6}, B_{3} \equiv A_{7}$, $C \equiv 2 A_{8}, \phi, \bar{\phi}$. Also, auxiliary fields are $\tilde{H}_{\mu}$ and $H_{\mathrm{A}}$. Scalars $\vec{B}, C$ arise via the dimensional reduction. Other fields $\psi_{ \pm \mu}, \vec{\chi}_{ \pm}$and $\eta_{ \pm}$are fermionic. The field contents are almost same as the two-dimensional $\mathcal{N}=4$ case except that each field in the quartet $\left(B, \chi_{ \pm}, H\right)$ is replaced with a three-component vector. For fields other than the quartet $\left(\vec{B}, \vec{\chi}_{ \pm}, \vec{H}\right)$, the $Q_{ \pm}$transformations (4.2) and (4.4) do not change. The quartet transforms as

$$
\begin{aligned}
& Q_{+} \vec{B}=\vec{\chi}_{+}, \quad Q_{+} \vec{\chi}_{+}=[\phi, \vec{B}], \\
& Q_{-} \vec{B}=\vec{\chi}_{-}, \quad Q_{-} \vec{\chi}_{-}=-[\bar{\phi}, \vec{B}], \\
& Q_{-} \chi_{+\mathrm{A}}=\frac{1}{2}\left[C, B_{\mathrm{A}}\right]-\frac{1}{2} \varepsilon_{\mathrm{ABC}}\left[B_{\mathrm{B}}, B_{\mathrm{C}}\right]-H_{\mathrm{A}}, \\
& Q_{+} \chi_{-\mathrm{A}}=\frac{1}{2}\left[C, B_{\mathrm{A}}\right]+\frac{1}{2} \varepsilon_{\mathrm{ABC}}\left[B_{\mathrm{B}}, B_{\mathrm{C}}\right]+H_{\mathrm{A}}, \\
& Q_{+} H_{\mathrm{A}}=\left[\phi, \chi_{-\mathrm{A}}\right]+\frac{1}{2}\left[B_{\mathrm{A}}, \eta_{+}\right]-\frac{1}{2}\left[C, \chi_{+\mathrm{A}}\right]-\varepsilon_{\mathrm{ABC}}\left[B_{\mathrm{B}}, \chi_{+\mathrm{C}}\right], \\
& Q_{-} H_{\mathrm{A}}=\left[\bar{\phi}, \chi_{+\mathrm{A}}\right]-\frac{1}{2}\left[B_{\mathrm{A}}, \eta_{-}\right]+\frac{1}{2}\left[C, \chi_{-\mathrm{A}}\right]-\varepsilon_{\mathrm{ABC}}\left[B_{\mathrm{B}}, \chi_{-\mathrm{C}}\right] .
\end{aligned}
$$

The nilpotency (4.5) is valid also here, and clearly seen is the $Q_{ \pm}$invariance of the action. With the same $\gamma$-matrix convention as in the $d=8$ case in appendix A, fermions (2.13) correspond to

$$
\Psi^{T}=\left(\psi_{+1}, \cdots, \psi_{+4},-\chi_{+1}, \chi_{+2}, \chi_{+3}, \frac{1}{2} \eta_{+}, \psi_{-1}, \cdots, \psi_{-4},-\chi_{-1}, \chi_{-2}, \chi_{-3}, \frac{1}{2} \eta_{-}\right) .
$$

$Q_{+}$is nothing but the dimensional reduction of $Q$ for the $\mathcal{N}=2, d=8$ case, and $Q_{-}$ represents the transformation $(2.6)$ with $\epsilon^{T}=(0,0,0,0,0,0,0,-\varepsilon, 0,0,0,0,0,0,0,0)$.

Enhancement of $\mathrm{U}(1)_{R}$ to $\mathrm{SU}(2)_{R}$ takes place, and the generators have the same form as in (4.8) with the straightforward modifications: $\mathrm{d}^{2} x \rightarrow \mathrm{d}^{4} x$ and $\chi_{ \pm}^{a}(x) \rightarrow \chi_{ \pm \mathrm{A}}^{a}(x)$. Also, the action (4.12) is invariant under $Q_{+} \leftrightarrow Q_{-}$with

$$
\begin{aligned}
& \phi \rightarrow-\bar{\phi}, \quad \bar{\phi} \rightarrow-\phi, \quad \vec{B} \rightarrow-\vec{B}, \\
& \vec{\chi}_{+} \rightarrow-\vec{\chi}_{-}, \quad \vec{\chi}_{-} \rightarrow-\vec{\chi}_{+}, \quad \tilde{H}_{\mu} \rightarrow-\tilde{H}_{\mu}, \\
& \psi_{ \pm \mu} \rightarrow \psi_{\mp \mu}, \quad \eta_{ \pm} \rightarrow \eta_{\mp} .
\end{aligned}
$$

\section{Lattice Formulation with Two Exact Supercharges}

Similarly to the $\mathcal{N}=2$ cases, we consider to define the theories (4.1), (4.12) on hypercubic lattices preserving the two supercharges $Q_{ \pm}$. Gauge fields $A_{\mu}$ are represented by the unitary variables $U_{\mu}(x)$ on the link $(x, x+\hat{\mu})$, while the other fields are put on sites.

For both cases of two- and four-dimensions, the transformation rule (4.2) is modified as

$$
Q_{+} U_{\mu}(x)=i \psi_{+\mu}(x) U_{\mu}(x)
$$




$$
\begin{aligned}
Q_{-} U_{\mu}(x)= & i \psi_{-\mu}(x) U_{\mu}(x) \\
Q_{+} \psi_{+\mu}(x)= & i \psi_{+\mu} \psi_{+\mu}(x)-i\left(\phi(x)-U_{\mu}(x) \phi(x+\hat{\mu}) U_{\mu}(x)^{\dagger}\right) \\
Q_{-} \psi_{-\mu}(x)= & i \psi_{-\mu} \psi_{-\mu}(x)+i\left(\bar{\phi}(x)-U_{\mu}(x) \bar{\phi}(x+\hat{\mu}) U_{\mu}(x)^{\dagger}\right) \\
Q_{-} \psi_{+\mu}(x)= & \frac{i}{2}\left\{\psi_{+\mu}(x), \psi_{-\mu}(x)\right\}-\frac{i}{2}\left(C(x)-U_{\mu}(x) C(x+\hat{\mu}) U_{\mu}(x)^{\dagger}\right)-\tilde{H}_{\mu}(x), \\
Q_{+} \psi_{-\mu}(x)= & \frac{i}{2}\left\{\psi_{+\mu}(x), \psi_{-\mu}(x)\right\}-\frac{i}{2}\left(C(x)-U_{\mu}(x) C(x+\hat{\mu}) U_{\mu}(x)^{\dagger}\right)+\tilde{H}_{\mu}(x), \\
Q_{+} \tilde{H}_{\mu}(x)= & -\frac{1}{2}\left[\psi_{-\mu}(x), \phi(x)+U_{\mu}(x) \phi(x+\hat{\mu}) U_{\mu}(x)^{\dagger}\right] \\
& +\frac{1}{4}\left[\psi_{+\mu}(x), C(x)+U_{\mu}(x) C(x+\hat{\mu}) U_{\mu}(x)^{\dagger}\right] \\
& +\frac{i}{2}\left(\eta_{+}(x)-U_{\mu}(x) \eta_{+}(x+\hat{\mu}) U_{\mu}(x)^{\dagger}\right) \\
& +\frac{i}{2}\left[\psi_{+\mu}(x), \tilde{H}_{\mu}(x)\right]+\frac{1}{4}\left[\psi_{+\mu}(x) \psi_{+\mu}(x), \psi_{-\mu}(x)\right] \\
Q_{-} \tilde{H}_{\mu}(x)= & -\frac{1}{2}\left[\psi_{+\mu}(x), \bar{\phi}(x)+U_{\mu}(x) \bar{\phi}(x+\hat{\mu}) U_{\mu}(x)^{\dagger}\right] \\
& -\frac{1}{4}\left[\psi_{-\mu}(x), C(x)+U_{\mu}(x) C(x+\hat{\mu}) U_{\mu}(x)^{\dagger}\right] \\
& -\frac{i}{2}\left(\eta_{-}(x)-U_{\mu}(x) \eta_{-}(x+\hat{\mu}) U_{\mu}(x)^{\dagger}\right) \\
& +\frac{i}{2}\left[\psi_{-\mu}(x), \tilde{H}_{\mu}(x)\right]-\frac{1}{4}\left[\psi_{-\mu}(x) \psi_{-\mu}(x), \psi_{+\mu}(x)\right] .
\end{aligned}
$$

The other transformations $((4.3,4.4)$ in two-dimensions, $(4.13,4.4)$ in four-dimensions) do not change the form under the latticization. Note that this modification keeps the nilpotency (4.5).

Making use of the $Q_{ \pm}$-transformation rule in terms of lattice variables, it is almost straightforward to construct lattice actions with the exact supercharges $Q_{ \pm}$for both of two- and four-dimensional cases:

$$
\begin{aligned}
S_{\mathcal{N}=4}^{2 d \mathrm{LAT}}=Q_{+} Q_{-} \frac{1}{2 g_{0}^{2}} \sum_{x} \operatorname{tr} & {\left[-i B(x) \Phi(x)-\sum_{\mu=1}^{2} \psi_{+\mu}(x) \psi_{-\mu}(x)-\chi_{+}(x) \chi_{-}(x)\right.} \\
& \left.-\frac{1}{4} \eta_{+}(x) \eta_{-}(x)\right], \\
S_{\mathcal{N}=4}^{4 d \mathrm{LAT}}=Q_{+} Q_{-} \frac{1}{2 g_{0}^{2}} \sum_{x} \operatorname{tr} & {\left[-i \vec{B}(x) \cdot \vec{\Phi}(x)-\frac{1}{3} \sum_{\mathrm{A}, \mathrm{B}, \mathrm{C}=1}^{3} \varepsilon_{\mathrm{ABC}} B_{\mathrm{A}}(x)\left[B_{\mathrm{B}}(x), B_{\mathrm{C}}(x)\right]\right.} \\
- & \left.\sum_{\mu=1}^{4} \psi_{+\mu}(x) \psi_{-\mu}(x)-\vec{\chi}_{+}(x) \cdot \vec{\chi}_{-}(x)-\frac{1}{4} \eta_{+}(x) \eta_{-}(x)\right],
\end{aligned}
$$

where $\Phi(x)$ and $\vec{\Phi}(x)$ are given by (3.7) and (3.8), respectively. The orders of operators on the lattice are

$$
\vec{B}(x), C(x), \phi(x), \bar{\phi}(x)=O(a), \quad \tilde{H}_{\mu}(x), \vec{H}(x)=O\left(a^{2}\right),
$$




$$
\text { (fermionic fields) }=O\left(a^{3 / 2}\right), \quad Q_{ \pm}=O\left(a^{1 / 2}\right) .
$$

Note that the lattice formulation retains the symmetries under $\mathrm{SU}(2)_{R}$ as well as the $Q_{+} \leftrightarrow Q_{-}$exchange (4.11 or 4.15). Generators of $\mathrm{SU}(2)_{R}$ take the same form as the continuum case under the trivial modification accompanied with the latticization $\left(\int \mathrm{d} x \rightarrow\right.$ $\sum_{x}$, etc). These symmetries are used later in argument for the renormalization.

\subsection{Two-dimensional Case}

Let us look closer the two-dimensional case (5.2), and discuss the renormalization.

After acting $Q_{ \pm}$, the action takes the form:

$$
\begin{aligned}
S_{\mathcal{N}=4}^{2 d \mathrm{LAT}}=\frac{1}{2 g_{0}^{2}} \sum_{x} \operatorname{tr}[ & -i\left(\frac{1}{2}[C(x), B(x)]+H(x)\right) \Phi(x)+H(x)^{2} \\
& +i \chi_{-}(x) Q_{+} \Phi(x)-i \chi_{+}(x) Q_{-} \Phi(x)-i B(x) Q_{+} Q_{-} \Phi(x) \\
& -[\phi(x), B(x)][\bar{\phi}(x), B(x)]-\frac{1}{4}[C(x), B(x)]^{2} \\
& +\chi_{+}(x)\left[\bar{\phi}(x), \chi_{+}(x)\right]-\chi_{-}(x)\left[\phi(x), \chi_{-}(x)\right]+\chi_{-}(x)\left[C(x), \chi_{+}(x)\right] \\
& -\chi_{-}(x)\left[B(x), \eta_{+}(x)\right]-\chi_{+}(x)\left[B(x), \eta_{-}(x)\right] \\
& -\frac{1}{4}[\phi(x), \bar{\phi}(x)]^{2}-\frac{1}{4}[\phi(x), C(x)][\bar{\phi}(x), C(x)] \\
& \left.-\frac{1}{4} \eta_{-}(x)\left[\phi(x), \eta_{-}(x)\right]+\frac{1}{4} \eta_{+}(x)\left[\bar{\phi}(x), \eta_{+}(x)\right]-\frac{1}{4} \eta_{+}(x)\left[C(x), \eta_{-}(x)\right]\right] \\
+\frac{1}{2 g_{0}^{2}} \sum_{x, \mu} \operatorname{tr} & {\left[\tilde{H}_{\mu}(x)^{2}-\frac{1}{2} \psi_{+\mu}(x) \psi_{+\mu}(x) \psi_{-\mu}(x) \psi_{-\mu}(x)\right.} \\
& +\left(\phi(x)-U_{\mu}(x) \phi(x+\hat{\mu}) U_{\mu}(x)^{\dagger}\right)\left(\bar{\phi}(x)-U_{\mu}(x) \bar{\phi}(x+\hat{\mu}) U_{\mu}(x)^{\dagger}\right) \\
& +\frac{1}{4}\left(C(x)-U_{\mu}(x) C(x+\hat{\mu}) U_{\mu}(x)^{\dagger}\right)^{2} \\
& -\psi_{+\mu}(x) \psi_{+\mu}(x)\left(\bar{\phi}(x)+U_{\mu}(x) \bar{\phi}(x+\hat{\mu}) U_{\mu}(x)^{\dagger}\right) \\
& +\psi_{-\mu}(x) \psi_{-\mu}(x)\left(\phi(x)+U_{\mu}(x) \phi(x+\hat{\mu}) U_{\mu}(x)^{\dagger}\right) \\
& -i \psi_{+\mu}(x)\left(\eta_{-}(x)-U_{\mu}(x) \eta_{-}(x+\hat{\mu}) U_{\mu}(x)^{\dagger}\right) \\
& -i \psi_{-\mu}(x)\left(\eta_{+}(x)-U_{\mu}(x) \eta_{+}(x+\hat{\mu}) U_{\mu}(x)^{\dagger}\right) \\
& \left.-\frac{1}{2}\left\{\psi_{+\mu}(x), \psi_{-\mu}(x)\right\}\left(C(x)+U_{\mu}(x) C(x+\hat{\mu}) U_{\mu}(x)^{\dagger}\right)\right] .
\end{aligned}
$$

Gauge kinetic terms appear from the $\Phi(x)^{2}$ term induced after $H(x)$ integrated out, whose structure is same as the $\mathcal{N}=2$ case. We add the supersymmetry breaking term (3.15) to single out the vacuum configuration $U_{\mu \nu}(x)=1$. $\tilde{H}_{\mu}(x)$ integrals yield kinetic terms of $B(x)$. Fermion kinetic terms come from the terms $\chi_{ \pm}(x) Q_{\mp} \Phi(x)$ and $\psi_{ \pm \mu}(x)\left(\eta_{\mp}(x)-\right.$ $\left.U_{\mu}(x) \eta_{\mp}(x+\hat{\mu}) U_{\mu}(x)^{\dagger}\right)$. The former has the same plaquette structure as in Fig. 1, and the species doublers are removed by the same mechanism ${ }^{7}$.

\footnotetext{
${ }^{7}$ The fermion kinetic terms take the same form as (3.16) with $P_{\mu}$ given by (B.5, B.6).
} 
We repeat the same renormalization argument as in the $\mathcal{N}=2, d=2$ case. The symmetry of the lattice action is sufficient to restore full supersymmetry and rotational invariance in the continuum limit. For instance, gauge invariance and $\mathrm{SU}(2)_{R}$ symmetry allow the operators $\operatorname{tr}\left(4 \phi \bar{\phi}+C^{2}\right)$ and $\operatorname{tr} B^{2}$, but they are not admissible from the supersymmetry $Q_{ \pm}$. For operators of the type $\varphi^{2}$, supersymmetry breaking effect by $\Delta S$ is irrelevant in two-dimensions. Thus, radiative corrections are not allowed to generate any relevant or marginal operators except the identity, which means that full supersymmetry and rotational invariance are restored in the continuum limit.

\subsection{Four-dimensional Case}

For the four-dimensional case, the plaquette structure of fermion kinetic terms is same, and fermion doublers are removed ${ }^{8}$. Although it is necessary to add some term like (3.15) in order to resolve the vacuum degeneracy, we will not discuss it here. In this subsection, we shall simply see how the exact supersymmetry in the action (5.3) works to restrict radiative corrections. The action (5.3) has a symmetry under the transformation $x \equiv$ $\left(x_{1}, x_{2}, x_{3}, x_{4}\right) \rightarrow \tilde{x} \equiv\left(x_{3}, x_{1}, x_{2}, x_{4}\right)$ (the cyclic permutation of $x_{1}, x_{2}, x_{3}$ with $x_{4}$ fixed) as

$$
\begin{aligned}
\left(U_{1}(x), U_{2}(x), U_{3}(x), U_{4}(x)\right) & \rightarrow\left(U_{2}(\tilde{x}), U_{3}(\tilde{x}), U_{1}(\tilde{x}), U_{4}(\tilde{x})\right), \\
\left(\psi_{ \pm 1}(x), \psi_{ \pm 2}(x), \psi_{ \pm 3}(x), \psi_{ \pm 4}(x)\right) & \rightarrow\left(\psi_{ \pm 2}(\tilde{x}), \psi_{ \pm 3}(\tilde{x}), \psi_{ \pm 1}(\tilde{x}), \psi_{ \pm 4}(\tilde{x})\right), \\
\left(\tilde{H}_{1}(x), \tilde{H}_{2}(x), \tilde{H}_{3}(x), \tilde{H}_{4}(x)\right) & \rightarrow\left(\tilde{H}_{2}(\tilde{x}), \tilde{H}_{3}(\tilde{x}), \tilde{H}_{1}(\tilde{x}), \tilde{H}_{4}(\tilde{x})\right) \\
\left(B_{1}(x), B_{2}(x), B_{3}(x)\right) & \rightarrow\left(B_{2}(\tilde{x}), B_{3}(\tilde{x}), B_{1}(\tilde{x})\right) \\
\left(\chi_{ \pm 1}(x), \chi_{ \pm 2}(x), \chi_{ \pm 3}(x)\right) & \rightarrow\left(\chi_{ \pm 2}(\tilde{x}), \chi_{ \pm 3}(\tilde{x}), \chi_{ \pm 1}(\tilde{x})\right), \\
\left(H_{1}(x), H_{2}(x), H_{3}(x)\right) & \rightarrow\left(H_{2}(\tilde{x}), H_{3}(\tilde{x}), H_{1}(\tilde{x})\right), \\
(\phi(x), C(x), \bar{\phi}(x)) & \rightarrow(\phi(\tilde{x}), C(\tilde{x}), \bar{\phi}(\tilde{x})), \\
\eta_{ \pm}(x) & \rightarrow \eta_{ \pm}(\tilde{x}) .
\end{aligned}
$$

The cyclic permutation of $x_{2}, x_{3}, x_{4}$ with $x_{1}$ fixed: $x \equiv\left(x_{1}, x_{2}, x_{3}, x_{4}\right) \rightarrow \tilde{x} \equiv\left(x_{1}, x_{4}, x_{2}, x_{3}\right)$ also keeps the action invariant:

$$
\begin{aligned}
\left(U_{1}(x), U_{2}(x), U_{3}(x), U_{4}(x)\right) & \rightarrow\left(U_{1}(\tilde{x}), U_{3}(\tilde{x}), U_{4}(\tilde{x}), U_{2}(\tilde{x})\right), \\
\left(\psi_{ \pm 1}(x), \psi_{ \pm 2}(x), \psi_{ \pm 3}(x), \psi_{ \pm 4}(x)\right) & \rightarrow\left(\psi_{ \pm 1}(\tilde{x}), \psi_{ \pm 3}(\tilde{x}), \psi_{ \pm 4}(\tilde{x}), \psi_{ \pm 2}(\tilde{x})\right), \\
\left(\tilde{H}_{1}(x), \tilde{H}_{2}(x), \tilde{H}_{3}(x), \tilde{H}_{4}(x)\right) & \rightarrow\left(\tilde{H}_{1}(\tilde{x}), \tilde{H}_{3}(\tilde{x}), \tilde{H}_{4}(\tilde{x}), \tilde{H}_{2}(\tilde{x})\right), \\
\left(B_{1}(x), B_{2}(x), B_{3}(x)\right) & \rightarrow\left(B_{3}(\tilde{x}),-B_{1}(\tilde{x}),-B_{2}(\tilde{x})\right), \\
\left(\chi_{ \pm 1}(x), \chi_{ \pm 2}(x), \chi_{ \pm 3}(x)\right) & \rightarrow\left(\chi_{ \pm 3}(\tilde{x}),-\chi_{ \pm 1}(\tilde{x}),-\chi_{ \pm 2}(\tilde{x})\right), \\
\left(H_{1}(x), H_{2}(x), H_{3}(x)\right) & \rightarrow\left(H_{3}(\tilde{x}),-H_{1}(\tilde{x}),-H_{2}(\tilde{x})\right), \\
(\phi(x), C(x), \bar{\phi}(x)) & \rightarrow(\phi(\tilde{x}), C(\tilde{x}), \bar{\phi}(\tilde{x})), \\
\eta_{ \pm}(x) & \rightarrow \eta_{ \pm}(\tilde{x}) .
\end{aligned}
$$

Thus, the action does not change under arbitrary permutations generated by (5.6) and (5.7), which contain $x \equiv\left(x_{1}, x_{2}, x_{3}, x_{4}\right) \rightarrow \tilde{x} \equiv\left(x_{4}, x_{2}, x_{1}, x_{3}\right)$ ( $x_{2}$ : fixed) and $x \equiv$ $\underline{\left(x_{1}, x_{2}, x_{3}, x_{4}\right) \rightarrow \tilde{x} \equiv\left(x_{4}, x_{1}, x_{3}, x_{2}\right)}\left(x_{3}\right.$ : fixed $)$.

\footnotetext{
${ }^{8}$ For the explicit form of the matrices $P_{\mu}$, see (B.5, B.7).
} 
In analysis on the renormalization, since the mass dimension of the coupling $g_{4}^{2}$ is zero, we should consider operators with $p \leq 4$ at all loop levels. For $p=1,2$, there exist no possible operators due to the gauge invariance, $\mathrm{SU}(2)_{R}$ and $Q_{ \pm}$supersymmetry. In $p=3$ case, it is convenient to use the result of $Q_{ \pm}$-cohomology in appendix C. According to the result (C.13), any gauge invariant and $\mathrm{SU}(2)_{R}$ invariant operators $\alpha$ annihilated by $Q_{ \pm}$ can be written as the form:

$$
\alpha=\alpha_{0}+Q_{+} \beta_{-}+Q_{+} Q_{-} \gamma
$$

with $\alpha_{0}$ proportional to the identity operator. From dimensional analysis and the degrees $(0,1)$ of $\beta_{-}$, the only possibility of the form of $\beta_{-}$is $A_{\mu} \psi_{-\nu}$. Since it can not be gauge invariant, the combination $Q_{+} \beta_{-}$does not exist. While the $Q_{+} \leftrightarrow Q_{-}$symmetry requires $\gamma$ being odd $(\gamma \rightarrow-\gamma)$, there is no such dimension-two operator with $\mathrm{SU}(2)_{R}$ invariance.

In the above, we saw that symmetries of the action (5.3) prevent the relevant operators with $p=1,2,3$ from being induced by loop corrections. For $p=4$ case, all the terms appearing in the action are marginal operators passing various symmetry requirements. The symmetries of the lattice action protect each structure of the following five terms against the quantum corrections:

$$
\begin{aligned}
& Q_{+} Q_{-} \operatorname{tr}[-i \vec{B} \cdot \vec{\Phi}], \quad Q_{+} Q_{-} \operatorname{tr}\left[\sum_{\mathrm{A}, \mathrm{B}, \mathrm{C}} \varepsilon_{\mathrm{ABC}} B_{\mathrm{A}}\left[B_{\mathrm{B}}, B_{\mathrm{C}}\right]\right], \quad Q_{+} Q_{-} \operatorname{tr}\left[\sum_{\mu} \psi_{+\mu} \psi_{-\mu}\right], \\
& Q_{+} Q_{-} \operatorname{tr}\left[\vec{\chi}_{+} \cdot \vec{\chi}_{-}\right], \quad Q_{+} Q_{-} \operatorname{tr}\left[\eta_{+} \eta_{-}\right],
\end{aligned}
$$

while relative weights among the five possibly would receive some corrections.

The subtlety on $p=4$ operators is specific in the four-dimensions. In the next two subsections, we will consider theories in lower dimensions obtained by dimensional reduction from the theory (5.3), and see two-dimensional model flowing to the desired continuum theories without fine tuning.

\section{$5.3 \mathcal{N}=8$ in Three-dimensions}

Dimensional reduction with respect to the $x^{4}$ direction from the action (5.3) leads the lattice action for three-dimensional $\mathcal{N}=8 \mathrm{SYM}$. Here, the dimensional reduction simply means to neglect the dependence on $x^{4}$ for all lattice variables. For example, the variable $U_{4}\left(x_{1}, x_{2}, x_{3}, x_{4}\right)$ on the link $(x, x+\hat{4})$ is reduced to the site variable $V\left(x_{1}, x_{2}, x_{3}\right)$ satisfying $V(x) V(x)^{\dagger}=1$. The form of the action (5.3) does not change, but now we should read

$$
U_{\mathrm{A} 4}(x)=U_{\mathrm{A}}(x) V(x+\hat{\mathrm{A}}) U_{\mathrm{A}}(x)^{\dagger} V(x)^{\dagger}, \quad U_{4 \mathrm{~A}}(x)=V(x) U_{\mathrm{A}}(x) V(x+\hat{\mathrm{A}})^{\dagger} U_{\mathrm{A}}(x)^{\dagger} .
$$

Also, we add the supersymmetry breaking term

$$
\Delta S=\frac{1}{2 g_{0}^{2}} \rho\left[\sum_{\mathrm{A}<\mathrm{B}} \operatorname{tr}\left(2-U_{\mathrm{AB}}(x)-U_{\mathrm{BA}}(x)\right)+\sum_{\mathrm{A}=1}^{3} \operatorname{tr}\left(2-U_{\mathrm{A} 4}(x)-U_{4 \mathrm{~A}}(x)\right)\right],
$$

where we consider on the $M \times M \times M$ periodic lattice, and the naive continuum limit is taken as $a \rightarrow 0$ with $g_{3}^{2} \equiv g_{0}^{2} / a$ and $L \equiv M a$ fixed. $\rho$ is chosen as $\rho=\frac{1}{M^{s}}=\frac{a^{s}}{L^{s}}$ 
with $0<s<1$ from the requirement that $\Delta S$ vanishes in the continuum limit while the levels of lifted vacua blow up. Under the dimensional reduction, symmetries of the four-dimensional model ( $\mathrm{SU}(2)_{R}, Q_{+} \leftrightarrow Q_{-}$symmetry (4.15) and the permutation (5.6)) persist in the three-dimensional model.

Now, let us go to the renormalization argument. We discuss on effect of the breaking term $\Delta S$ later. In three-dimensions, the mass dimension of the coupling $g_{3}^{2}$ is one. Operators of the type $\varphi^{a} \partial^{b} \psi^{2 c}$ with the dimension $p=a+b+3 c$ receive loop corrections as

$$
\left(\frac{a^{p-4}}{g_{3}^{2}}+c_{1} a^{p-3}+c_{2} a^{p-2} g_{3}^{2}+c_{3} a^{p-1} g_{3}^{4}+c_{4} a^{p} g_{3}^{6}+\cdots\right) \int \mathrm{d}^{3} x \varphi^{a} \partial^{b} \psi^{2 c},
$$

where the notations are similar as in (3.21). From the formula, we read that operators with $p \leq 4-\ell$ are relevant or marginal at the $\ell$-loop level. We can use the list in Table 1 .

For operators with $p=1,2$, gauge invariance, $\mathrm{SU}(2)_{R}$ and $Q_{+} \leftrightarrow Q_{-}$symmetry allow

$$
\operatorname{tr}\left(4 \phi \bar{\phi}+C^{2}\right), \quad \operatorname{tr}\left(A_{4}^{2}\right), \quad \operatorname{tr}\left(B_{1}^{2}+B_{2}^{2}+B_{3}^{2}\right), \quad \operatorname{tr}\left(B_{1} B_{2}+B_{2} B_{3}+B_{3} B_{1}\right),
$$

while all of them are forbidden by the $Q_{ \pm}$supersymmetry. In order to see the $p=3$ operators, we use the result of $Q_{ \pm}$-cohomology (C.13). From dimensional analysis, candidates for $\beta_{-}$are $\operatorname{tr}\left(A_{4} \psi_{-\mu}\right)$, but they are not $Q_{-}$invariant. Hence there is no operator of the type $Q_{+} \beta_{-}$. Symmetries under $Q_{+} \leftrightarrow Q_{-}$and the permutation $\left(x_{1}, x_{2}, x_{3}\right) \rightarrow\left(x_{3}, x_{1}, x_{2}\right)$ reduce candidates for $\gamma$ to $\operatorname{tr}\left(\left(B_{1}+B_{2}+B_{3}\right) A_{4}\right)$ alone.

Loop diagrams with $n$ vertices of $\Delta S$ inserted induce supersymmetry breaking effect, which is suppressed by the factor $\frac{a^{n s}}{L^{n s}}$ for each loop contribution in (5.12). The power of ' $a$ ' for the $p=2$ operators at the one-loop is $a^{n s-1}$, so the operators (5.13) remain relevant for $n=1$ and can not be excluded. On the other hand, the $p=3$ operators becomes irrelevant when $\Delta S$-vertices inserted.

Therefore, the radiative corrections are allowed to generate the operator $Q_{+} Q_{-} \operatorname{tr}\left(\left(B_{1}+\right.\right.$ $\left.\left.B_{2}+B_{3}\right) A_{4}\right)$ and those in (5.13). In order to reach the desired supersymmetric continuum theory, we have to add counter terms for the operators and cancel them with the radiative corrections by tuning parameters.

\section{$5.4 \mathcal{N}=8$ in Two-dimensions}

As a result of further dimensional reduction with respect to $x^{3}$, we obtain a lattice theory for $\mathcal{N}=8$ in two-dimensions. In addition to the site variable $V(x)$ coming from $U_{4}(x)$, there appears a new site variable $W(x)$, which arises from $U_{3}(x)$, satisfying $W(x) W(x)^{\dagger}=1$. The form of the action (5.3) is still valid with

$$
\begin{aligned}
& U_{\mu 4}(x)=U_{4 \mu}(x)^{\dagger}=U_{\mu}(x) V(x+\hat{\mu}) U_{\mu}(x)^{\dagger} V(x)^{\dagger}, \\
& U_{\mu 3}(x)=U_{3 \mu}(x)^{\dagger}=U_{\mu}(x) W(x+\hat{\mu}) U_{\mu}(x)^{\dagger} W(x)^{\dagger}, \\
& U_{34}(x)=U_{43}(x)^{\dagger}=W(x) V(x) W(x)^{\dagger} V(x)^{\dagger} .
\end{aligned}
$$


Here, $\mu=1,2$ for gauge variables. Similar to the previous cases, we consider the $M \times M$ periodic lattice and the supersymmetry breaking term

$$
\Delta S=\frac{1}{2 g_{0}^{2}} \rho \sum_{x} \sum_{1 \leq k<l \leq 4} \operatorname{tr}\left(2-U_{k l}(x)-U_{l k}(x)\right),
$$

with $\rho=\frac{1}{M^{s}}=\frac{a^{s}}{L^{s}}(0<s<2)$.

From the same renormalization argument, it is seen that loop corrections are not allowed to generate any relevant or marginal operators except the identity, which means restoration of full supersymmetry and rotational invariance in the continuum limit.

\section{Summary and Discussions}

In this paper, $\mathrm{SU}(N)$ super Yang-Mills theories with extended supersymmetry have been constructed on hyper-cubic lattices of various dimensions, keeping one or two supercharges exactly. Our lattice models have a huge degeneracy of the classical vacua, which does not allow the standard argument based on the lattice perturbation theory. In order to avoid the difficulty, we added to the action some supersymmetry breaking term, which becomes invisible in the naive continuum limit. Among the lattice models, the desired continuum theories are obtained without any fine tuning of parameters for the cases $\mathcal{N}=2,4,8$ in two-dimensions. We have arrived at the conclusion checking that symmetries of the lattice action forbid radiative corrections to generate any relevant or marginal operators which prevent the lattice theory from flowing to the continuum theory. It is interesting to see the restoration of full supersymmetry and rotational invariance explicitly by computer simulation.

To resolve the vacuum degeneracy, it would be also possible to impose the admissibility condition on each plaquette variable:

$$
\left\|1-U_{\mu \nu}(x)\right\|<\epsilon
$$

similar to that for gauge fields coupled to the Ginsparg-Wilson fermions [20]. It breaks the supersymmetry picking up contribution from the boundary $\left\|1-U_{\mu \nu}(x)\right\|=\epsilon$, but the breaking effect would vanish in the weak coupling limit. It will be interesting to pursue the possibility and to try reformulating our models in terms of the Ginsparg-Wilson fermions.

It is also worth to be investigated the dynamics of our lattice actions without the supersymmetry breaking terms, nonperturbative understanding of which will be necessary to identify the corresponding continuum theories.

The lattice actions have no symmetry under discrete rotations by 90 degrees due to the specific couplings between fermions $\chi$ and $\psi$. It is possible to modify the couplings keeping the supersymmetry so that the theories are symmetric under the 90-degree rotations. At the same time, however doublers arise from both of bosonic and fermionic sectors, as expected from the manifest supersymmetry. It is interesting to identify the corresponding continuum theories for both cases with and without the supersymmetry breaking terms.

While we have focussed on pure Yang-Mills theories, it is possible to extend our formulation to the case of matter fields coupled, since the actions of $\mathcal{N}=2,4$ supersymmetric QCD are rewritten as 'TFT and BTFT forms' [21]. 


\section{Acknowledgments}

The author would like to thank H. Fukaya, Y. Kikukawa, T. Onogi and T. Takimi for valuable discussions, and M. Fukuma, S. Iso, H. Kunitomo, M. Ninomiya, S. Ohta and S-J. Rey for useful comments. He also thank the theory group members of KEK, YITP and RIKEN for their warm hospitality during his stay. Finally, he would like to thank Y. Shamir for crucial comments on the first version of this paper.

\section{A. Explicit Form of $\gamma_{i}$ for $\mathcal{N}=2$}

Here, we present the explicit form of the matrices $\gamma_{i}$ employed for 'TFT form' of $\mathcal{N}=2$ SYM theories. $\gamma_{i}$ is written as the form

$$
\gamma_{i}=-i\left(\begin{array}{cc}
0 & \mu_{i} \\
\mu_{i}^{T} & 0
\end{array}\right) \quad \text { for } i=1, \cdots, D-2, \quad \gamma_{D-1}=i \sigma_{3} \otimes \mathbf{1}_{D-2},
$$

where $\sigma_{1}, \sigma_{2}, \sigma_{3}$ denote the Pauli matrices, and the explicit forms of $\mu_{i}$ are as follows.

For $d=2$,

$$
\mu_{1}=\sigma_{1}, \quad \mu_{2}=-\sigma_{3} .
$$

For $d=4$,

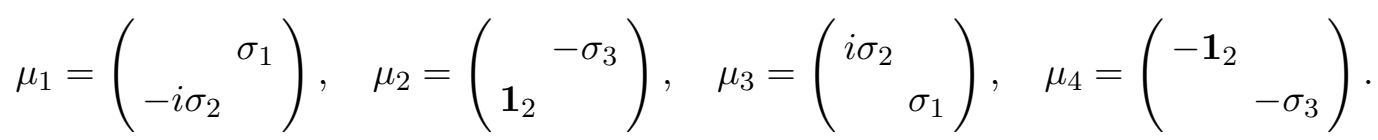

For $d=8$,

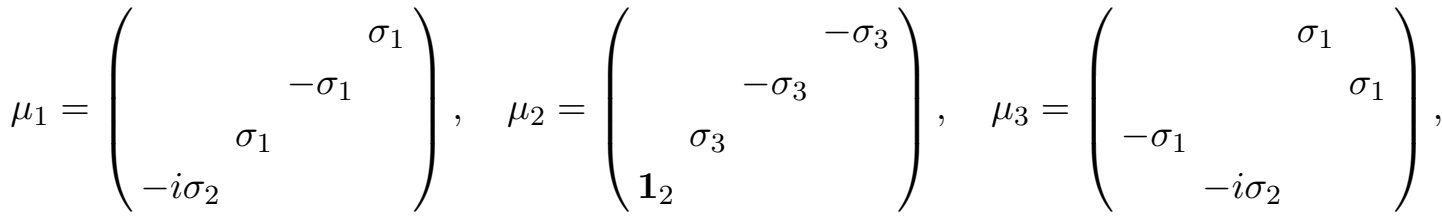

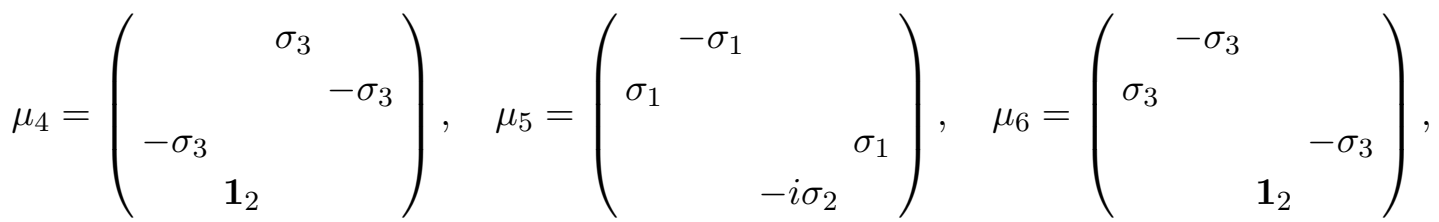

$$
\begin{aligned}
& \mu_{7}=\left(\begin{array}{cccc}
i \sigma_{2} & & & \\
& i \sigma_{2} & & \\
& & i \sigma_{2} & \\
& & & \sigma_{1}
\end{array}\right), \quad \mu_{8}=\left(\begin{array}{llll}
-\mathbf{1}_{2} & & & \\
& -\mathbf{1}_{2} & & \\
& & -\mathbf{1}_{2} & \\
& & & -\sigma_{3}
\end{array}\right) \text {. }
\end{aligned}
$$

The cases $d=4,8$ correspond also to 'BTFT form' for $\mathcal{N}=4$ SYM theories in twoand four-dimensions, respectively. 


\section{B. Explicit Form of $P_{\mu}$ for $\mathcal{N}=2,4$}

For both cases $\mathcal{N}=2$ and $\mathcal{N}=4$, fermion kinetic terms in the lattice actions are expressed as the form (3.16). We present the explicit form of matrices $P_{\mu}$ appearing in the expressions.

In the $\mathcal{N}=2$ theories, $P_{\mu}$ are written as the form

$$
P_{\mu}=\left(\begin{array}{cc}
0 & \nu_{\mu} \\
\nu_{\mu} & 0
\end{array}\right)=\sigma_{1} \otimes \nu_{\mu} \quad \text { for } \mu=1, \cdots, d-1, \quad P_{d}=\sigma_{2} \otimes \mathbf{1}_{d} .
$$

For each case of $d=2,4,8, \nu_{\mu}$ are given as follows.

For $d=2$ case,

$$
\nu_{1}=\sigma_{2}
$$

For $d=4$ case,

$$
\nu_{1}=\sigma_{1} \otimes \sigma_{2}, \quad \nu_{2}=\sigma_{2} \otimes \mathbf{1}_{2}, \quad \nu_{3}=-\sigma_{3} \otimes \sigma_{2} .
$$

For $d=8$ case,

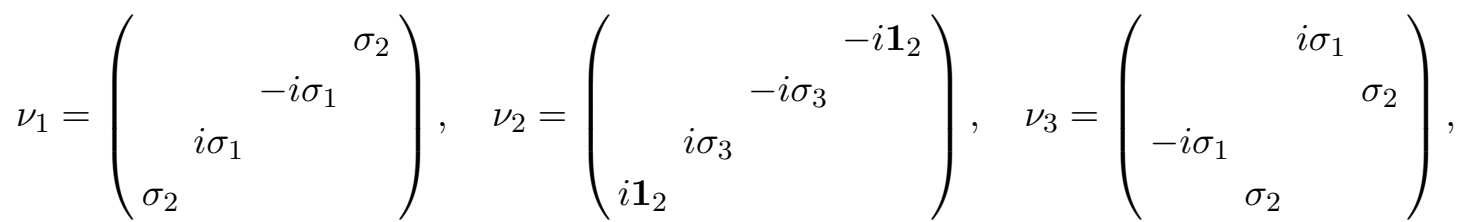

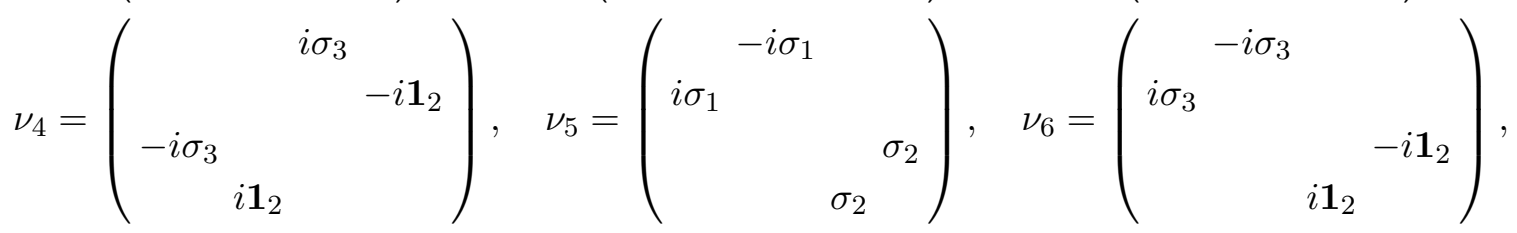

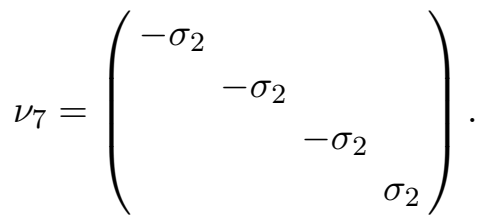

In the $\mathcal{N}=4$ theories, $P_{\mu}$ are expressed as

$$
P_{\mu}=-i\left(\begin{array}{cc}
0 & \omega_{\mu} \\
-\omega_{\mu}^{T} & 0
\end{array}\right)
$$

where the explicit forms of $\omega_{\mu}$ are presented as below.

For two-dimensional case,

$$
\omega_{1}=\left(\begin{array}{r}
i \sigma_{2} \\
-\sigma_{1}
\end{array}\right), \quad \omega_{2}=\left(\begin{array}{c}
\mathbf{1}_{2} \\
\sigma_{3}
\end{array}\right) .
$$

For four-dimensional case,

$$
\omega_{1}=\left(\begin{array}{c}
{ }^{2}{ }^{i \sigma_{2}} \\
\sigma_{1}
\end{array}\right.
$$




$$
\omega_{4}=\left(\begin{array}{cccc} 
& & -\sigma_{3} & \\
& & & \mathbf{1}_{2} \\
-\sigma_{3} & & & \\
& & & \\
& & &
\end{array}\right) \text {. }
$$

\section{C. $Q_{ \pm}$-cohomology on Lattice}

In this appendix, we discuss on the equivalent cohomology based on two supercharges $Q_{+}$, $Q_{-}$nilpotent up to gauge transformations in the theory $(5.3)^{9}$. The basic fact is given in Theorem 2.1 in ref. [14], and this gives a generalization in the context of lattice gauge theory.

First, we assign the degrees $\left(q_{+}, q_{-}\right)$to each variable as follows:

$$
\begin{array}{ccc}
U_{\mu}:(0,0) & \vec{B}:(1,1) & \phi:(2,0) \\
\psi_{+\mu}:(1,0) & \vec{\chi}_{+}:(2,1) & \bar{\phi}:(0,2) \\
\psi_{-\mu}:(0,1) & \vec{\chi}_{-}:(1,2) & C:(1,1) . \\
\tilde{H}_{\mu}:(1,1) & \vec{H}:(2,2) & \eta_{+}:(2,1) \\
& & \eta_{-}:(1,2)
\end{array}
$$

The charges $q_{ \pm}$are measured by the operators:

$$
\begin{aligned}
& L_{+}=\sum_{x, a}\left[\psi_{+\mu}^{a}(x) \frac{\partial}{\partial \psi_{+\mu}^{a}(x)}+\tilde{H}_{\mu}^{a}(x) \frac{\partial}{\partial \tilde{H}_{\mu}^{a}(x)}+B_{\mathrm{A}}^{a}(x) \frac{\partial}{\partial B_{\mathrm{A}}^{a}(x)}+2 \chi_{+\mathrm{A}}^{a}(x) \frac{\partial}{\partial \chi_{+\mathrm{A}}^{a}(x)}\right. \\
& +\chi_{-\mathrm{A}}^{a}(x) \frac{\partial}{\partial \chi_{-\mathrm{A}}^{a}(x)}+2 H_{\mathrm{A}}^{a}(x) \frac{\partial}{\partial H_{\mathrm{A}}^{a}(x)}+C^{a}(x) \frac{\partial}{\partial C^{a}(x)}+2 \phi^{a}(x) \frac{\partial}{\partial \phi^{a}(x)} \\
& \left.+2 \eta_{+}^{a}(x) \frac{\partial}{\partial \eta_{+}^{a}(x)}+\eta_{-}^{a}(x) \frac{\partial}{\partial \eta_{-}^{a}(x)}\right], \\
& L_{-}=\sum_{x, a}\left[\psi_{-\mu}^{a}(x) \frac{\partial}{\partial \psi_{-\mu}^{a}(x)}+\tilde{H}_{\mu}^{a}(x) \frac{\partial}{\partial \tilde{H}_{\mu}^{a}(x)}+B_{\mathrm{A}}^{a}(x) \frac{\partial}{\partial B_{\mathrm{A}}^{a}(x)}+2 \chi_{-\mathrm{A}}^{a}(x) \frac{\partial}{\partial \chi_{-\mathrm{A}}^{a}(x)}\right. \\
& +\chi_{+\mathrm{A}}^{a}(x) \frac{\partial}{\partial \chi_{+\mathrm{A}}^{a}(x)}+2 H_{\mathrm{A}}^{a}(x) \frac{\partial}{\partial H_{\mathrm{A}}^{a}(x)}+C^{a}(x) \frac{\partial}{\partial C^{a}(x)}+2 \bar{\phi}^{a}(x) \frac{\partial}{\partial \bar{\phi}^{a}(x)} \\
& \left.+2 \eta_{-}^{a}(x) \frac{\partial}{\partial \eta_{-}^{a}(x)}+\eta_{+}^{a}(x) \frac{\partial}{\partial \eta_{+}^{a}(x)}\right],
\end{aligned}
$$

where ' $a$ ' is the index with respect to the gauge group generators, and the summation over the indices $\mu$ and $\mathrm{A}$ is assumed. The $\mathrm{U}(1)_{R}$ charge is given by $q_{+}-q_{-}$and $J_{0}=L_{+}-L_{-}$. $Q_{ \pm}$and $J_{ \pm \pm}$shift the degrees as

$$
\begin{aligned}
Q_{+}:\left(q_{+}, q_{-}\right) \rightarrow\left(q_{+}+1, q_{-}\right), & Q_{-}:\left(q_{+}, q_{-}\right) \rightarrow\left(q_{+}, q_{-}+1\right), \\
J_{++}:\left(q_{+}, q_{-}\right) \rightarrow\left(q_{+}+1, q_{-}-1\right), & J_{--}:\left(q_{+}, q_{-}\right) \rightarrow\left(q_{+}-1, q_{-}+1\right) .
\end{aligned}
$$

\footnotetext{
${ }^{9}$ The argument is valid also for the two-dimensional case (5.2) with reading the three-component vectors $\vec{B}, \vec{\chi}_{ \pm}, \vec{H}$ as the one-component $B, \chi_{ \pm}, H$.
} 
Next, it is convenient for later argument to introduce the 'homotopy operators' $K_{ \pm}$:

$$
\begin{aligned}
K_{+}=-\sum_{x, a} & {\left[\psi_{+\mu}^{a}(x) \frac{\partial}{\partial \tilde{H}_{\mu}^{a}(x)}+2 \chi_{+\mathrm{A}}^{a}(x) \frac{\partial}{\partial H_{\mathrm{A}}^{a}(x)}-B_{\mathrm{A}}^{a}(x) \frac{\partial}{\partial \chi_{-\mathrm{A}}^{a}(x)}+2 \phi^{a}(x) \frac{\partial}{\partial \eta_{+}^{a}(x)}\right.} \\
& \left.-C^{a}(x) \frac{\partial}{\partial \eta_{-}^{a}(x)}\right], \\
K_{-}=\sum_{x, a} & {\left[\psi_{-\mu}^{a}(x) \frac{\partial}{\partial \tilde{H}_{\mu}^{a}(x)}+2 \chi_{-\mathrm{A}}^{a}(x) \frac{\partial}{\partial H_{\mathrm{A}}^{a}(x)}+B_{\mathrm{A}}^{a}(x) \frac{\partial}{\partial \chi_{+\mathrm{A}}^{a}(x)}+2 \bar{\phi}^{a}(x) \frac{\partial}{\partial \eta_{-}^{a}(x)}\right.} \\
+ & \left.C^{a}(x) \frac{\partial}{\partial \eta_{+}^{a}(x)}\right],
\end{aligned}
$$

with changing the degrees as

$$
K_{+}:\left(q_{+}, q_{-}\right) \rightarrow\left(q_{+}, q_{-}-1\right), \quad K_{-}:\left(q_{+}, q_{-}\right) \rightarrow\left(q_{+}-1, q_{-}\right) .
$$

Since there is no operator with negative $q_{ \pm}$, for any operator $\alpha$ with $\left(q_{+}, q_{-}\right)$

$$
\begin{array}{ll}
J_{++} \alpha=K_{+} \alpha=0 & \text { if } q_{-}=0, \\
J_{--} \alpha=K_{-} \alpha=0 & \text { if } q_{+}=0 .
\end{array}
$$

After some calculation, it turns out that the following algebra holds:

$$
\begin{aligned}
& \left\{Q_{+}, K_{+}\right\}=-J_{++}, \quad\left\{Q_{-}, K_{+}\right\}=L_{+}, \\
& \left\{Q_{-}, K_{-}\right\}=-J_{--}, \quad\left\{Q_{+}, K_{-}\right\}=L_{-},
\end{aligned}
$$

which plays a key role for the argument on $Q_{ \pm}$-cohomology below.

Suppose that $\alpha$ is a gauge invariant and $\mathrm{SU}(2)_{R}$ invariant operator with the degrees $\left(q_{+}, q_{-}\right)$, and that $Q_{ \pm}$annihilate $\alpha: Q_{ \pm} \alpha=0$. Since $K_{-} Q_{+} \alpha=0$, making use of (C.7) leads

$$
\alpha=\left\{\begin{array}{cl}
Q_{+}\left(\frac{1}{q_{-}} K_{-} \alpha\right) & \text { for } q_{+} \geq 1, q_{-} \geq 1 \\
0 & \text { for } q_{+}=0, q_{-} \geq 1
\end{array}\right.
$$

When $q_{-}=0, Q_{+}$-cohomology can be nontrivial. Similarly, starting with $K_{+} Q_{-} \alpha=0$, we arrive at

$$
\alpha=\left\{\begin{array}{cl}
Q_{-}\left(\frac{1}{q_{+}} K_{+} \alpha\right) & \text { for } q_{+} \geq 1, q_{-} \geq 1 \\
0 & \text { for } q_{+} \geq 1, q_{-}=0
\end{array}\right.
$$

Nontrivial $Q_{-}$-cohomology can appear when $q_{+}=0$.

Let us consider the case $q_{+} \geq 1, q_{-} \geq 1$. Note that $K_{-} \alpha$ is annihilated by $Q_{-}$as a consequence of (C.7). Thus, by the same argument to (C.8),

$$
K_{-} \alpha=Q_{-}\left(\frac{1}{q_{+}-1} K_{+} K_{-} \alpha\right) \quad \text { for } q_{+} \geq 2, q_{-} \geq 1
$$

Plugging this into (C.8) leads

$$
\alpha=Q_{+} Q_{-}\left(\frac{1}{q_{-}\left(q_{+}-1\right)} K_{+} K_{-} \alpha\right) \quad \text { for } q_{+} \geq 2, q_{-} \geq 1 .
$$


From the similar consideration on $K_{+} \alpha$, we obtain

$$
\alpha=Q_{-} Q_{+}\left(\frac{1}{q_{+}\left(q_{-}-1\right)} K_{-} K_{+} \alpha\right) \quad \text { for } q_{+} \geq 1, q_{-} \geq 2 \text {. }
$$

In the case $q_{+}=q_{-}=0, \alpha$ is a function of $U_{\mu}$. But, from the condition of $Q_{ \pm}$-closed, it must be proportional to the identity operator. Finally, when $q_{+}=q_{-}=1, \alpha$ is expressed as $\alpha=Q_{+}\left(K_{-} \alpha\right)=Q_{-}\left(K_{+} \alpha\right)$, where $K_{ \pm} \alpha$ are $Q_{ \pm}$-closed and expanded by $\psi_{ \pm \mu}$, respectively.

The above result is summarized as the following statement:

Any gauge invariant and $S U(2)_{R}$ invariant operator $\alpha$ annihilated by $Q_{ \pm}$can be expressed as

$$
\begin{aligned}
\alpha & =\alpha_{0}+Q_{+} \beta_{-}+Q_{+} Q_{-} \gamma \\
& =\alpha_{0}+Q_{-} \beta_{+}+Q_{+} Q_{-} \gamma
\end{aligned}
$$

$\alpha_{0}$ is proportional to the identity, and $\beta_{ \pm}, \gamma$ are gauge invariant. $\beta_{+}\left(\beta_{-}\right)$is a $Q_{+^{-}}\left(Q_{-_{-}}\right)$ closed operator of the degrees $(1,0)((0,1))$, and has nontrivial cohomology with respect to $Q_{+}\left(Q_{-}\right)$.

The argument is valid also for lattice SYM theories obtained by dimensional reduction from (5.3), for instance $\mathcal{N}=8$ theories in two- and three-dimensions.

\section{References}

[1] N. Seiberg and E. Witten, Electric - magnetic duality, monopole condensation, and confinement in N=2 supersymmetric Yang-Mills theory, Nucl. Phys. B 426 (1994) 19 [Erratum-ibid. B 430 (1994) 485] [hep-th/9407087]; Monopoles, duality and chiral symmetry breaking in N=2 supersymmetric QCD, Nucl. Phys. B 431 (1994) 484 [hep-th/9408099].

[2] J. M. Maldacena, The large N limit of superconformal field theories and supergravity, Adv. Theor. Math. Phys. 2 (1998) 231 [Int. J. Theor. Phys. 38 (1999) 1113] [hep-th/9711200]. O. Aharony, S. S. Gubser, J. M. Maldacena, H. Ooguri and Y. Oz, Large N field theories, string theory and gravity, Phys. Rept. 323 (2000) 183 [hep-th/9905111].

[3] T. Banks and P. Windey, Supersymmetric Lattice Theories, Nucl. Phys. B 198 (1982) 226.

I. Ichinose, Supersymmetric Lattice Gauge Theory, Phys. Lett. B 122 (1983) 68.

J. Bartels and J. B. Bronzan, Supersymmetry On A Lattice, Phys. Rev. D 28 (1983) 818.

J. Bartels and G. Kramer, A Lattice Version Of The Wess-Zumino Model, Z. Phys. C 20 (1983) 159.

R. Nakayama and Y. Okada, Supercurrent Anomaly In Lattice Gauge Theory, Phys. Lett. B 134 (1984) 241.

S. Nojiri, Continuous 'Translation' And Supersymmetry On The Lattice, Prog. Theor. Phys. $\mathbf{7 4}$ (1985) 819.

G. Curci and G. Veneziano, Supersymmetry And The Lattice: A Reconciliation?, Nucl. Phys. B 292 (1987) 555.

K. Fujikawa, Supersymmetry on the lattice and the Leibniz rule, Nucl. Phys. B 636 (2002) 80 [hep-th/0205095]. 
[4] S. Elitzur, E. Rabinovici and A. Schwimmer, Supersymmetric Models On The Lattice, Phys. Lett. B 119 (1982) 165.

[5] D. B. Kaplan, Dynamical Generation Of Supersymmetry, Phys. Lett. B 136 (1984) 162. J. Nishimura, Four-dimensional $N=1$ supersymmetric Yang-Mills theory on the lattice without fine-tuning, Phys. Lett. B 406 (1997) 215 [hep-lat/9701013].

N. Maru and J. Nishimura, Lattice formulation of supersymmetric Yang-Mills theories without fine-tuning, Int. J. Mod. Phys. A 13 (1998) 2841 [hep-th/9705152].

H. Neuberger, Vector like gauge theories with almost massless fermions on the lattice, Phys. Rev. D 57 (1998) 5417 [hep-lat/9710089].

D. B. Kaplan and M. Schmaltz, Supersymmetric Yang-Mills theories from domain wall fermions, Chin. J. Phys. 38 (2000) 543 [hep-lat/0002030].

G. T. Fleming, J. B. Kogut and P. M. Vranas, Super Yang-Mills on the lattice with domain wall fermions, Phys. Rev. D 64 (2001) 034510 [hep-lat/0008009].

I. Montvay, Supersymmetric Yang-Mills theory on the lattice, Int. J. Mod. Phys. A 17 (2002) 2377 [hep-lat/0112007].

[6] S. Cecotti and L. Girardello, Stochastic Processes In Lattice (Extended) Supersymmetry, Nucl. Phys. B 226 (1983) 417.

N. Sakai and M. Sakamoto, Lattice Supersymmetry And The Nicolai Mapping, Nucl. Phys. B 229 (1983) 173.

S. Catterall and S. Karamov, Exact lattice supersymmetry: the two-dimensional $N=2$ Wess-Zumino model, Phys. Rev. D 65 (2002) 094501 [hep-lat/0108024].

Y. Kikukawa and Y. Nakayama, Nicolai mapping vs. exact chiral symmetry on the lattice, Phys. Rev. D 66 (2002) 094508 [hep-lat/0207013].

K. Fujikawa, $N=2$ Wess-Zumino model on the $d=2$ Euclidean lattice, Phys. Rev. D 66 (2002) 074510 [hep-lat/0208015].

[7] D. B. Kaplan, E. Katz and M. Ünsal, Supersymmetry on a spatial lattice, JHEP 0305 (2003) 037 [hep-lat/0206019].

[8] A. G. Cohen, D. B. Kaplan, E. Katz and M. Ünsal, Supersymmetry on a Euclidean spacetime lattice. I: A target theory with four supercharges, JHEP 0308 (2003) 024 [hep-lat/0302017]; Supersymmetry on a Euclidean spacetime lattice. II: Target theories with eight supercharges, [hep-lat/0307012].

[9] J. Nishimura, S. J. Rey and F. Sugino, Supersymetry on the noncommutative lattice, JHEP 0302 (2003) 032 [hep-lat/0301025].

J. Giedt, E. Poppitz and M. Rozali, Deconstruction, lattice supersymmetry, anomalies and branes, JHEP 0303 (2003) 035 [hep-th/0301048].

J. Giedt, Non-positive fermion determinants in lattice supersymmetry, Nucl. Phys. B 668 (2003) 138 [hep-lat/0304006]; The fermion determinant in $(4,4)$ 2d lattice super-Yang-Mills, [hep-lat/0307024].

[10] W. Bietenholz, Exact supersymmetry on the lattice, Mod. Phys. Lett. A 14 (1999) 51 [hep-lat/9807010].

K. Itoh, M. Kato, H. Sawanaka, H. So and N. Ukita, Novel approach to super Yang-Mills theory on lattice: Exact fermionic symmetry and 'Ichimatsu' pattern, JHEP 0302 (2003) 033 [hep-lat/0210049]. 
S. Catterall and S. Karamov, A lattice study of the two-dimensional Wess Zumino model, Phys. Rev. D 68 (2003) 014503 [hep-lat/0305002].

[11] S. Catterall, Lattice supersymmetry and topological field theory, JHEP 0305 (2003) 038 [hep-lat/0301028].

[12] E. Witten, Topological Quantum Field Theory, Commun. Math. Phys. 117 (1988) 353; Introduction To Cohomological Field Theories, Int. J. Mod. Phys. A 6 (1991) 2775.

[13] C. Vafa and E. Witten, A Strong coupling test of $S$ duality, Nucl. Phys. B 431 (1994) 3 [hep-th/9408074].

[14] R. Dijkgraaf and G. W. Moore, Balanced topological field theories, Commun. Math. Phys. 185 (1997) 411 [hep-th/9608169].

[15] M. Blau and G. Thompson, Aspects of $N(T) \geq 2$ topological gauge theories and D-branes, Nucl. Phys. B 492 (1997) 545 [hep-th/9612143].

J. M. F. Labastida and C. Lozano, Mathai-Quillen formulation of twisted $N=4$ supersymmetric gauge theories in four dimensions, Nucl. Phys. B 502 (1997) 741 [hep-th/9702106].

[16] P. van Nieuwenhuizen and A. Waldron, On Euclidean spinors and Wick rotations, Phys. Lett. B 389 (1996) 29 [hep-th/9608174].

M. Blau and G. Thompson, Euclidean SYM theories by time reduction and special holonomy manifolds, Phys. Lett. B 415 (1997) 242 [hep-th/9706225].

[17] R. Brooks, D. Montano and J. Sonnenschein, Gauge Fixing And Renormalization In Topological Quantum Field Theory, Phys. Lett. B 214 (1988) 91.

[18] L. Baulieu, H. Kanno and I. M. Singer, Special quantum field theories in eight and other dimensions, Commun. Math. Phys. 194 (1998) 149 [hep-th/9704167].

[19] H. B. Nielsen and M. Ninomiya, Absence Of Neutrinos On A Lattice. 1. Proof By Homotopy Theory, Nucl. Phys. B 185 (1981) 20 [Erratum-ibid. B 195 (1982) 541]; Absence Of Neutrinos On A Lattice. 2. Intuitive Topological Proof, Nucl. Phys. B 193 (1981) 173.

[20] P. H. Ginsparg and K. G. Wilson, A Remnant Of Chiral Symmetry On The Lattice, Phys. Rev. D 25 (1982) 2649.

P. Hasenfratz, Prospects for perfect actions, Nucl. Phys. Proc. Suppl. 63 (1998) 53 [hep-lat/9709110].

H. Neuberger, Exactly massless quarks on the lattice, Phys. Lett. B 417 (1998) 141 [hep-lat/9707022]; More about exactly massless quarks on the lattice, Phys. Lett. B 427 (1998) 353 [hep-lat/9801031].

P. Hernandez, K. Jansen and M. Lüscher, Locality properties of Neuberger's lattice Dirac operator, Nucl. Phys. B 552 (1999) 363 [hep-lat/9808010].

[21] M. Alvarez and J. M. F. Labastida, Topological matter in four-dimensions, Nucl. Phys. B 437 (1995) 356 [hep-th/9404115].

S. Hyun, J. Park and J. S. Park, Spin-c Topological QCD, Nucl. Phys. B 453 (1995) 199 [hep-th/9503201].

A. Sako and T. Sasaki, Euler number of instanton moduli space and Seiberg-Witten invariants, J. Math. Phys. 42 (2001) 130 [hep-th/0005262]. 